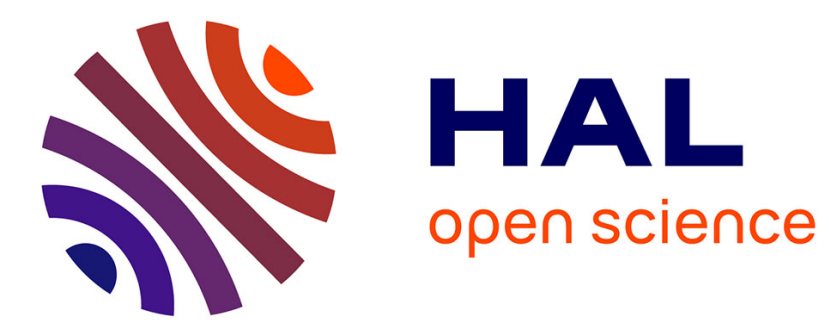

\title{
L'impact de la pression temporelle sur le traitement des informations
}

\author{
Jeanne Lallement
}

\section{To cite this version:}

Jeanne Lallement. L'impact de la pression temporelle sur le traitement des informations. Recherche et Applications en Marketing (French Edition), 2010, 25 (4), pp.45-68. 10.1177/076737011002500403 . hal-01488477

\section{HAL Id: hal-01488477 \\ https://hal.science/hal-01488477}

Submitted on 9 Jan 2018

HAL is a multi-disciplinary open access archive for the deposit and dissemination of scientific research documents, whether they are published or not. The documents may come from teaching and research institutions in France or abroad, or from public or private research centers.
L'archive ouverte pluridisciplinaire HAL, est destinée au dépôt et à la diffusion de documents scientifiques de niveau recherche, publiés ou non, émanant des établissements d'enseignement et de recherche français ou étrangers, des laboratoires publics ou privés. 


\title{
L'impact de la pression temporelle sur le traitement des informations
}

\author{
Jeanne Lallement \\ Maître de conférences \\ IUT TC La Rochelle \\ Centre de recherche $L R M O S-C E R E G E$
}

RÉSUMÉ

L'objectif principal de cette recherche est d'étudier les effets de la pression temporelle sur le traitement des informations. Pour rendre compte du processus de décision en situation de temps contraint, une expérimentation a été réalisée par la méthode informatisée des tables d'informations auprès de 521 consommateurs d'ordinateurs portables. Il en ressort que l'intensité de la pression temporelle a une influence non linéaire sur la vitesse de traitement des informations, induit une sélection des attributs les plus importants, et ne modifie que légèrement la méthode de recherche d'information adoptée par le consommateur. Le traitement de la pression temporelle en trois niveaux (nulle, modérée, forte) conforte l'idée d'un processus adaptatif non linéaire entre la pression temporelle et les variables dépendantes.

Mots clés : Pression temporelle, comportement du consommateur, temps, traitement des informations. 


\section{INTRODUCTION}

La pression temporelle est une réalité sociale. $24 \%$ des Français sont décrits par Sécodip comme des « chrono victimes » ${ }^{1}$. Souvent « dépassés par les événements, ils culpabilisent de ne réussir à gérer leur temps ». Les « chrono actifs », débordant d'obligations familiales, professionnelles ou sociales représentent $15 \%$ des individus sondés. Leurs préoccupations majeures sont de concilier au mieux les différentes activités composant leur « budget temps ». Les années 2000 sont marquées par « l'accélération du temps ». L'urbanisation et l'industrialisation sont à l'origine d'une conscience accrue d'un manque de temps. À la ressource temporelle finie (24 heures par jour) s'ajoutent de nombreuses obligations (manger, dormir, travailler). Dans ce contexte, cette recherche traite des conséquences de ce sentiment de manque de temps sur le comportement de traitement des informations.

En marketing, de trop rares recherches ont étudié l'influence du temps contraint sur les étapes cognitives du traitement de l'information. Les auteurs reconnaissent les formidables capacités d'adaptation des consommateurs à la pression temporelle. Un consensus théorique se dégage, considérant trois effets majeurs de la pression temporelle sur le traitement des informations. La pression temporelle entraîne : 1) une accélération de la vitesse de traitement de chaque information, 2) une sélection des éléments d'informations considérés, 3) un changement de la stratégie décisionnelle pour s'adapter à la contrainte temporelle. En dépit de cette convergence, des questions subsistent sur les modalités d'adaptation de ces mécanismes par le consommateur en fonction de l'intensité de cette pression temporelle. La vitesse de traitement est-elle fonction de l'intensité de la pression temporelle exercée ? Sur quels critères la sélection se réalise-t-elle ? Quels liens ces trois mécanismes entretiennent-ils entre eux ? Récemment, des recherches ont suggéré de classer la pression temporelle selon trois niveaux (sans pres- sion temporelle, pression modérée et pression forte), remplaçant alors l'ancienne distinction, avec ou sans pression temporelle (Suri et Monroe, 2003 ; ChienHuang et Pei-Hsun, 2005). Tel est l'objectif de cette étude, grâce à une application originale de la méthode des tables d'information, permettant d'éclairer le caractère linéaire ou non de la pression temporelle dans les mécanismes d'accélération et de sélection.

Au-delà des enjeux théoriques liés à la prise de décision, l'étude des effets de la pression temporelle ouvre trois perspectives importantes sur le plan méthodologique, conceptuel et managérial. Tout d'abord, cette recherche propose une méthodologie originale, mise en place à partir d'une adaptation informatisée et en ligne de la méthode des tables d'information, qui permet d'interroger un échantillon conséquent de consommateurs. En second lieu, les études antérieures n'ont pas approfondi les conséquences liées à l'intensité de la pression temporelle. Or, l'examen de son intensité permet de renouveler et d'approfondir les connaissances sur les effets de la pression temporelle. Enfin, le troisième apport vise à souligner l'importance pour les distributeurs de considérer précisément la contrainte temporelle des consommateurs afin de proposer une réponse adaptée.

La pression temporelle est une question d'actualité qui soulève des questions théoriques fondamentales. Les recherches sur le sujet restent imprécises. Un des buts de cet article est de circonscrire le concept de pression temporelle et ses conséquences sur le traitement des informations. Cet objectif nous permettra alors d'atteindre deux autres buts : 1) dresser un inventaire des recherches et des expérimentations assistées par ordinateur s'intéressant à la pression temporelle 2) proposer une adaptation informatisée des tables d'information ouvrant de nouvelles perspectives théoriques et méthodologiques. 


\section{FONDEMENTS CONCEPTUELS ET HYPOTHÈSES}

\section{La pression temporelle : définitions}

On retrouve dans la plupart des médias populaires des thèmes autour de l'accélération de notre mode de vie, la civilisation de l'urgence ou encore la recherche de gain de temps à tout prix. Depuis trente ans, le manque de temps disponible est également un thème omniprésent dans la presse managériale et académique, avec des développements d'inspiration économique centrés sur le temps de travail et d'autres plus sociologiques reliant ce phénomène aux variables socio-économiques explicatives (la famille, le genre, la profession, l'éducation). Les recherches en sciences humaines centrées sur la pression temporelle, essentiellement anglo-saxonnes, témoignent de par la terminologie utilisée de la richesse du concept mais aussi de son manque de consensus. Les termes employés s'organisent autour de deux notions : un manque de temps objectif (déficit de temps, manque de temps, rareté du temps, famine temporelle, temps contraint $^{2}$ ) et le sentiment qui l'accompagne (temps accéléré, temps rapide, urgence, accélération du temps ${ }^{3}$ ). Le terme de pression temporelle choisi dans cette recherche se justifie par cette double approche.

Concevoir la pression temporelle à partir du temps quantitatif, mesurable et divisible, permet d'expliciter la notion. Dans l'approche d'origine économique (Linder, 1970 ; Voss et Blackwell, 1975), le temps est segmenté selon les occupations humaines (travail, activités obligatoires et loisirs). La pression temporelle correspond alors à un temps discrétionnaire insuffisant, contraint par une ressource naturelle finie. Pour les chercheurs, traiter de la pression temporelle revient à reconnaître l'incongruence temporelle expérimentée par les individus (Hendrix et Martin, 1981 ; Mantel et Kellaris, 2003; Sullivan, 2008). Il s'agit d'un problème d'allocation de temps, selon l'expression des sociologues (Feldman et Hornik, 1981 ; Cotte et Ratneshwar, 1998 ; Reeves et

2. Time deficit, time poverty, time scarcity, time famine, time squeeze.

3. Speed up time, fast time, urgency, accelerated pace of life.
Szafran, 1996), qui s'exprime à tous les stages du processus de décision (Nickols et Fox, 1983 ; Berry, 1979 ; Okada et Hoch, 2004 ; Herrington et Capella, 1995), qui peut être objectivement mesuré (Hornik, 1984 ; Hendrix, 1984 ; Park, Iyer et Smith, 1989 ; Robinson et Nicosia, 1991 ; Marmorstein, 1992 ; Kellaris et Mantel, 1994) et peut même être contrôlé (Kolodinsky, 1990 ; Nelmapius et alii, 2005).

Parallèlement, les termes utilisés soulignent l'aspect expérientiel et affectif de la pression temporelle, qui ne saurait se réduire à une simple cognition (Hawes, 1980 ; Bergadaà, 1988 ; Kaufman et Lane, 1990). Il s'agit d'une expérience individuelle, appréciée subjectivement par chacun. Les travaux sur les temps d'attente s'inscrivent dans cette thématique de la subjectivité dans le jugement temporel (Zakay, 2005). L'expérience de la durée est hautement dépendante du contexte dans lequel il prend place, des caractéristiques du participant et de ses activités (Hornik, 1993 ; Kang, Herr et Page, 2003 ; Leclerc, Schmitt et Dubé, 1995 ; Comm et Palachek, 1984 ; Hui, Tse et Zhou, 2006 ; Durrande-Moreau, 1994). Dans les travaux d'inspiration psychologique, le temps s'étudie sous l'angle de son appréhension par les consciences individuelles (Bergson, 1922). La conception individuelle du temps, l'orientation temporelle ${ }^{4}$ correspond alors à une force motivationnelle qui influence le comportement du consommateur (Urien, 1994; Bergadaà, 1989, 1988 ; ValetteFlorence et alii, 2001 ; Usunier et Valette-Florence, 2007 ; Urien, 2007) et le choix de sa formule d'achat (Cotte et Ligas, 2003; Bouder-Pailler, 2003 ; Bergadaà et Coraux, 2004 ; Bergadaà, 2005). Dès lors, la pression temporelle est appréciée différemment selon l'orientation temporelle et le caractère mono ou polychrone ${ }^{5}$ des individus (Koiso-Kanttila, 2005). En outre, Bergadaà (2007), en proposant quatre types de « temps-cadres » structurant du comportement humain, identifie deux dimensions temporelles particulièrement en lien avec un sentiment de pression temporelle chronique : le « temps présent

\footnotetext{
4. Bergadaà (1989) définit l'orientation temporelle comme la prédisposition de l'individu à se visualiser dans une des trois zones temporelles (passé, présent, futur).

5. Le style monochrone désigne les individus qui traitent les informations de façon séquentielle. Par opposition, les polychrones se singularisent par leur capacité à réaliser plusieurs tâches en même temps (Hall, 1959).
} 
fragmenté », où l'urgence devient le modèle de référence absolue, et le «temps rythmé » qui concilie l'urgence et le temps choisi.

Deux notions conceptuelles différentes sont précisément regroupées sous le terme de pression temporelle. De façon quotidienne, le temps peut exercer une force qui contraint les individus à agir de manière prompte, c'est le «temps présent fragmenté » présenté précédemment (Bergadaà, 2007) que l'on pourrait rapprocher de la pression temporelle chronique mesurée dans certains travaux (Aho, 2007 ; Rizkalla, 1989 ; Ackerman et Gross, 2003). Par opposition à l'essence perpétuelle de cette contrainte temporelle (Kim et Kim, 2008 ; Szollos, 2009), le sujet de recherche porte sur la pression temporelle situationnelle qui s'exerce de façon ponctuelle. L'origine de la contrainte temporelle permet de clarifier la notion (Wright et Weitz, 1977). Darpy (1999) propose une classification des échéances selon un axe éluctable/inéluctable, soulignant l'intensité de la pression et les possibilités de procrastination du consommateur. D'autres recherches classent les échéances en fonction de leur origine, distinguant les échéances internes, choisies par l'individu, des causes externes et notamment « sociétales » suggérées par l'environnement (Miyazaki, 1993 ; Gross, 1994 ; Aggarwal et Vaidyanathan, 2003 ; Abendroth et Diehl, 2006 ; Brannon et Brock, 2001 ; Ariely et Wertenbroch, 2002). À l'instar de la plupart des travaux en marketing consacrés à l'influence de la pression temporelle sur le processus de décision, le choix a été fait d'utiliser le terme de pression temporelle ${ }^{6}$. Il ne s'agit ni de l'urgence, supposant une action dans la précipitation (Riveline, 1991 ; Aubert, 2003 ; Usunier, 1995), ni du fait "d'être pressé », où la forme passive suggère une action involontaire, subie et un sentiment négatif, mais bien de l'appréciation individuelle d'avoir ponctuellement un temps insuffisant pour réaliser une tâche. Quelle que soit l'origine de l'échéance, celle-ci limite les capacités de l'individu à considérer entièrement les informations pour prendre sa décision de façon optimale (Park, Iyer et Smith, 1989 ; Schellinck, 1983 ; Pieters, Warlop et Hartog, 1997 ; Dhar et Nowlis, 1999 ; Suri et Monroe, 2003).

6. Time pressure.

\section{Les conséquences de la pression temporelle sur le traitement des informations}

La plupart des recherches sur les effets de la pression temporelle s'inscrivent dans un courant cognitif et analysent les conséquences de la contrainte temporelle sur le processus rationnel de traitement des informations. Cette recherche propose de préciser selon l'intensité de la pression temporelle les trois mécanismes de coping, mis en avant par Miller (1960) : l'accélération du rythme décisionnel, la sélection des informations et la modification de la méthode qui permet d'examiner les informations.

Le premier mécanisme développé par l'individu pour faire face à la contrainte temporelle est un changement d'intensité dans la vitesse de traitement de l'information. Ce processus est nommé l'accélération. L'individu tente de répondre à la contrainte temporelle en traitant un nombre identique d'informations, mais avec une vitesse supérieure. De nombreux auteurs ont fait état de ce processus d'accélération du traitement de l'information (Maule, Hockey et Bdzola, 2000 ; Bettman, Luce et Payne, 1998 ; Payne, Bettman et Johnson, 1988). Avec le deuxième mécanisme, l'individu ne traite plus qu'une partie de l'information disponible, ce qui lui simplifie la décision. La revue de littérature identifie deux stratégies différentes. Certains (Edland, 1993 ; Kerstholt, 1994 ; Payne, Bettman et Johnson, 1988) mettent l'accent sur un processus de choix conscient de certains attributs par le consommateur. Ils utilisent alors le terme de sélection. D'autres font l'hypothèse d'un mécanisme moins conscient, qui donne plus d'importance aux informations négatives (Wright et Weitz, 1977). Le terme employé est alors celui de filtrage, ou filtration, selon l'intérêt porté au processus ou au résultat. Les consommateurs veulent éviter les mauvaises options et l'approche des résultats du choix rend les menaces plus saillantes (Jewel, 2003).

Le troisième mécanisme d'adaptation à la pression temporelle décrit un changement de règle décisionnelle. Lorsque la tâche décisionnelle se complique et que la modification de l'intensité de la règle de décision ne suffit plus, les individus peuvent changer de méthode d'examen des informations. Deux changements de stratégie de décision sont le plus souvent décrits : le passage d'un mode compensatoire à un mode non compensatoire et la stratégie d'évitement. Selon Bettman, Luce et Payne (1998), 
plus la pression temporelle est forte et plus les individus adoptent des règles de décision fondées sur l'examen comparatif des attributs (« les stratégies intra-attributs ») au détriment de règles décisionnelles comparant deux à deux des alternatives complètes (« les stratégies intra-alternatives »). En effet, lorsque le temps est insuffisant pour un examen exhaustif des informations (où tous les attributs sont évalués), alors les individus passent d'une stratégie fondée sur la profondeur (détaillant les offres) à une stratégie plus simple reposant sur la largeur (les attributs). Jacoby et alii (1994) précisent que les stratégies intra-attributs diminuent la sensation d'incertitude des consommateurs de façon significative. D'autre part, il existe de nombreuses situations réelles où le consommateur peut différer son choix. La décision de non-choix et le report du choix, l'ajournement, font l'objet d'un courant de recherche croissant (Chien-Huang et Pei-Hsun, 2005 ; Arts, 2000 ; Dhar et Nowlis, 1999 ; Darpy, 1999 ; Dhar, 1997 ; Greenleaf et Lehmann, 1995). En synthèse de ces travaux, il apparaît que la difficulté issue d'une pression temporelle grandissante peut favoriser l'ajournement et que cet effet est contingent de l'attractivité de la décision et du type de décision (Dhar et Nowlis, 1999). Le Tableau 1 propose une synthèse des recherches consacrées à ces trois effets de la pression temporelle sur le processus de décision. Les répercussions de la pression temporelle sur l'accélération et la sélection sont vérifiées dans la majeure partie des recherches. En revanche, l'action de la pression sur le changement de stratégie décisionnel est plus controversée.

\section{Impact de l'intensité de la pression temporelle sur le traitement des informations}

Au-delà de ce consensus apparent, des interrogations subsistent dans la littérature sur le rôle que joue l'intensité de la pression temporelle sur les modalités d'applications de ces trois mécanismes (Weenig et Maarleveld, 2002 ; Maule, Hockey et Bdzola, 2000). Ainsi, si le principe d'accélération est vérifié dans une vingtaine de recherches répertoriées, deux travaux le contredisent. Les résultats de Weenig et Maarleveld (2002) témoignent d'un contexte particulier, où la pression temporelle suscite un examen plus long de chaque information donnée. Concrètement, leurs sujets, soumis à un stress temporel, passent 7 secondes en moyenne sur chaque caractéristique de l'offre, là où les consommateurs sans contrainte de temps ne restent que 5 secondes. Selon les auteurs, leurs résultats sont en lien avec la complexité de la tâche, et l'accélération n'est possible que dans un contexte particulier de faible activité cognitive, avec des tâches simples. Dans cette même lignée, Topi, Valacich et Hoffer (2005) montrent que dans une situation de tâche complexe, le processus d'accélération ne se vérifie plus. Avec un examen approfondi des études antérieures, on se rend compte que l'impact de la pression temporelle n'a été testé que pour un seul degré d'intensité de pression, et uniquement dans un contexte de tâche simple, variant sur un nombre restreint d'attributs. En s'attachant à détailler les conséquences de l'intensité de la pression temporelle sur l'accélération, cette recherche postule l'existence d'effets non linéaires. Lorsque la pression temporelle est modérée, le consommateur peut augmenter sa vitesse de traitement de chaque information pour répondre à sa contrainte temporelle, c'est l'accélération. Par contre, en situation de forte contrainte temporelle, là où la complexité est accrue, la forte activité cognitive déployée empêche l'accélération. Le consommateur réduit alors sa vitesse moyenne de traitement de chaque attribut.

\section{H1: Les consommateurs passent plus de temps sur chaque élément d'information lorsque la pression temporelle est modérée que lorsque celle-ci est nulle ou forte. ${ }^{7}$}

Pour prendre sa décision, le consommateur dispose de nombreuses informations, correspondant aux différentes offres. Chacune de ces options se caractérise par un ensemble d'attributs, tels que la marque, le prix, les caractéristiques techniques. La deuxième hypothèse propose de vérifier le principe selon lequel les individus sous pression temporelle utilisent globalement moins d'informations pour prendre leur décision, et que cette sélection s'opère de façon graduelle sur le nombre global d'informations disponibles (H2a), mais aussi sur le nombre d'attributs (H2b) et le nombre d'offres (H2c) considérés.

7. L'hypothèse $\mathrm{H} 1$ et les suivantes nécessitent de suivre les recommandations méthodologiques de Suri et Monroe (2003) qui suggèrent une manipulation de différents niveaux d'intensité de la pression temporelle. Cette particularité méthodologique sera éclairée ultérieurement. 


\begin{tabular}{|c|c|c|c|c|c|c|c|c|c|c|c|c|c|c|c|c|c|c|c|c|}
\hline \multicolumn{2}{|c|}{$\begin{array}{l}\text { Les effets sur le processus de } \\
\text { traitement de l'information }\end{array}$} & 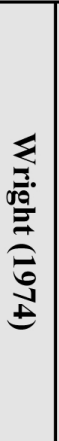 & 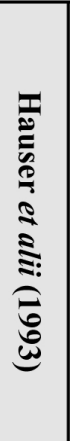 & 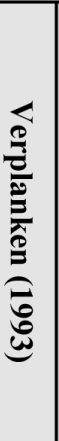 & 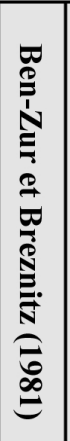 & 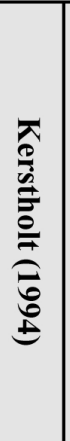 & 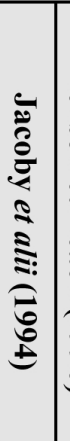 & 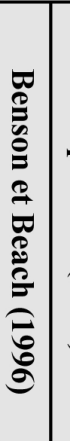 & 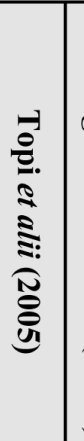 & 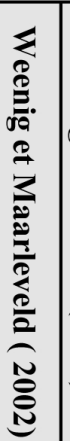 & 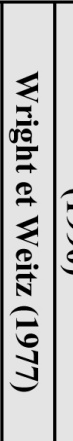 & 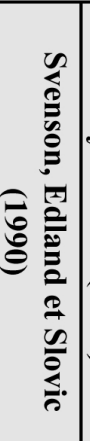 & 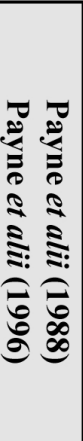 & 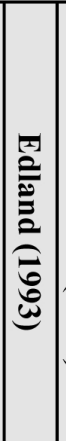 & 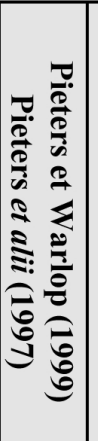 & 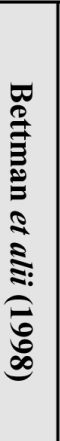 & 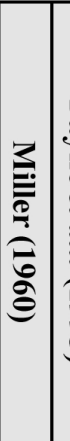 & 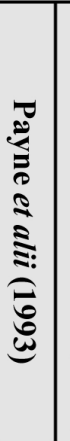 & 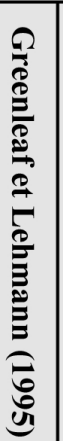 & 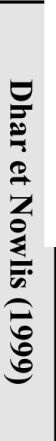 \\
\hline 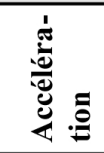 & $\begin{array}{l}\text { Processus d'accélération confirmé } \\
(+) \text { ou infirmé }(-)\end{array}$ & + & + & & + & + & & & - & - & + & & + & & + & + & & & & \\
\hline \multirow[b]{2}{*}{ 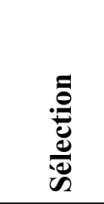 } & Processus de filtration & + & + & + & + & & & & & + & + & & + & + & + & + & & & & \\
\hline & Processus de sélection & + & + & + & + & + & & & & + & + & & + & + & + & + & & & & \\
\hline \multirow{4}{*}{ 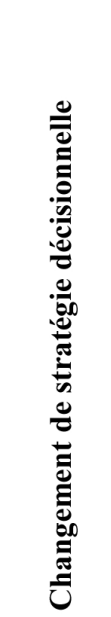 } & $\begin{array}{l}\text { Changement de } \\
\text { stratégie décisionnelle confirmé } \\
(+) \text { ou infirmé }\end{array}$ & & & & + & - & + & - & & - & + & + & + & & + & + & & & & \\
\hline & $\begin{array}{l}\text { Passage du mode compensatoire à } \\
\text { un mode non compensatoire } \\
\text { confirmé }(+) \text { ou infirmé }(-)\end{array}$ & & & & & & & & & & + & - & + & + & + & & & & & \\
\hline & $\begin{array}{l}\text { Passage d'un processus par } \\
\text { alternative à un processus par } \\
\text { attribut }\end{array}$ & & & & & & & & & & & & & + & + & + & & & & \\
\hline & $\begin{array}{l}\text { Processus d'ajournement } \\
\text { confirmé (+) ou infirmé (-) }\end{array}$ & & & & & & & & & & & & & & & & & + & + & - \\
\hline
\end{tabular}


H2 : Plus la pression temporelle est forte, plus a) le nombre global d'informations examinées diminue ; b) le nombre d'attributs examinés par le consommateur diminue; c) le nombre d'offres examinées par le consommateur diminue.

L'hypothèse $\mathrm{H} 3$ propose de préciser la manière dont s'opère la sélection. Deux écoles s'opposent. Edland et Svenson (1993), à la suite d'une synthèse des études empiriques réalisées, concluent qu'il existe une relation linéaire entre l'importance des attributs et le niveau d'attention. Ainsi, les attributs les moins importants aux yeux du consommateur « disparaissent » en premier dans l'examen des informations. En revanche, les caractéristiques les plus importantes de l'offre paraissent plus robustes face à la pression. Même si la pression temporelle est forte, ces déterminants de l'offre restent prioritairement étudiés par le consommateur. Pour Weenig et Maarleveld (2002), par contre, la sélectivité s'opère surtout aux dépens des attributs modérés. Face à la difficulté de certaines tâches, l'individu peut négliger les attributs de faible importance. Cependant, la pression temporelle entraîne une concentration des efforts sur les informations vitales. Dès lors, l'augmentation de l'intensité de la pression temporelle décrit une relation curviligne, où l'effet de la sélectivité s'exerce de façon plus forte sur les attributs d'importance moyenne que sur ceux de forte ou de faible importance. Dans leurs travaux (Weenig et Maarleveld, 2002), les attributs modérés sont plus sévèrement «touchés » par la pression temporelle. Pourtant les auteurs justifient assez peu théoriquement ces résultats. Dès lors l'hypothèse $H 3$, en accord avec les premiers travaux mentionnés (Edland et Svenson, 1993), suppose que la sélection s'opère de façon logique, en accordant plus d'importance aux attributs vitaux.

H3 : Plus la pression temporelle est forte, plus le processus de sélection s'exerce sur les attributs considérés d'importance faible comparés aux attributs de moyenne et de forte importance.

Les règles de décisions non compensatoires sont particulièrement adaptées au contexte de pression temporelle. Pour mesurer le passage à une stratégie en largeur plutôt qu'en profondeur, Payne (1976) propose de comparer, dans la succession des infor- mations consultées par le consommateur, le nombre de transitions entre deux attributs de la même offre avec le nombre total de transitions entre deux mêmes attributs pour des offres différentes. Le score, appelé « score de Payne ${ }^{8}$, indique s'il est négatif une prédominance de recherche intra-attributs ou, s'il est positif, un plus grand nombre de règles décisionnelles reposant sur une comparaison approfondie des offres. L'hypothèse $\mathrm{H} 4$ suppose que la pression temporelle suscite une baisse des stratégies comparant des options entre elles et, inversement, une augmentation des règles décisionnelles non compensatoires de type intra-attributs.

\section{H4 : Plus la pression temporelle est forte, plus la proportion de stratégies fondées sur des comparaisons d'attributs augmente, plus la proportion de stratégies fondées sur des comparaisons des offres complètes baisse.}

Selon les auteurs, les trois mécanismes ci-dessus l'accélération, la sélection et le changement de règle décisionnelle - sont décrits comme additifs, compensatoires ou complémentaires. Ben-Zur et Breznitz (1981) supposent une accumulation des différentes réactions à la pression temporelle. Bettman, Luce et Payne (1998) fondent leur théorie sur un système hiérarchique, lié à l'intensité de la pression temporelle, mais ne le testent pas. Maule, Hockey et Bdzola (2000) supposent un mécanisme de compensation entre les processus d'accélération et de sélection, en fonction du style cognitif des individus. Il n'y a pas de consensus sur le sujet. Dès lors, l'hypothèse $\mathrm{H} 5$, à l'instar des travaux les plus récents de Maule, Hockey et Bdzola (2000), suppose que l'accélération et la sélection sont deux mécanismes substituables. Le consommateur peut adapter sa stratégie décisionnelle par des effets d'accélération ou de sélection, et les deux mécanismes se compensent.

\section{H5 : Dans un contexte de pression temporelle, une forte accélération est associée à une plus faible sélection, et inversement, une faible accélération correspond à une forte sélection.}

8. Son mode de calcul est explicité page 11. 
Des travaux récents (Chien-Huang et Pei-Hsun, 2005) supposent l'existence d'une stratégie décisionnelle « confuse » lorsque la pression temporelle est forte. Il n'existe pas, à ce jour, de travaux vérifiant l'effet d'une forte pression temporelle sur ces changements de règles décisionnelles. Seul le passage d'un mode décisionnel en profondeur à un mode en largeur, centré sur les attributs, a été mesuré dans des recherches antérieures. Or, le sujet peut également développer une stratégie moins rationnelle, plus « aléatoire ». La stratégie décisionnelle " aléatoire » est définie dans cet article par une recherche d'information qui n'est pas fondée sur un ordre logique. Par opposition avec les stratégies intra-attributs ou intraalternatives qui utilisent des informations directement comparables deux à deux, la stratégie décisionnelle confuse est décrite par une consultation d'informations dans un ordre aléatoire (deux informations sans lien direct entre elles). L'hypothèse H6 propose de vérifier l'effet de l'intensité de la pression temporelle sur le choix de ce mode décisionnel.

H6 : Plus la pression temporelle est forte, plus le nombre de stratégies décisionnelles aléatoires, fondées sur la consultation d'informations deux à deux sans lien entre elles, augmente.

\section{Intérêts de la méthode informatisée des tables d'information}

L'intérêt de l'utilisation de l'IDB pour cette recherche est double. En premier lieu, elle répond aux objectifs assignés à cette recherche, puisqu'il s'agit d'une des rares méthodes permettant d'appréhender la nature dynamique du processus décisionnel, avec, de surcroît, une manipulation précise de la variable temps et une procédure strictement identique pour tous les sujets. En second lieu, compte tenu des possibilités offertes par les nouvelles technologies, la version informatique de la procédure offre des nouvelles possibilités facilitant sa mise en œuvre et le traitement des données. Très récemment, les travaux de Petr et Hess-Miglioretti (2010) ont montré la robustesse de la méthode auto-administrée sur Internet. La méthode des tables d'information obéit à une procédure simple. On présente au consommateur un certain nombre d'informations et on analyse la façon dont celui-ci les utilise. Concrètement, le sujet est soumis à un tableau à double entrée : en ligne figurent les différentes offres ; en colonne, celles-ci sont décrites par des attributs. Le tableau 2 présente les modalités d'application choisies dans les trop rares recherches utilisant la méthode.

\section{Variables dépendantes}

\section{MÉTHODOLOGIE}

Les études traitant des effets de la pression temporelle ont majoritairement adopté des approches expérimentales et notamment la méthode des tables d'information. Celle-ci, connue sous le nom d'IDB (Information Display Board) aux États-Unis, a été développée par Jacoby en 1978 puis popularisée en France par Dubois (1984). Elle permet de comprendre la façon dont le consommateur utilise et traite l'information pour aboutir à un choix final. Bien que la méthode des tables d'information soit reprise dans de nombreux ouvrages faisant référence $^{9}$, il n'existe que peu d'applications.

9. Evrard Y., Pras B. et Roux E. (1993), Market: Études et recherches en marketing, Paris, Nathan, pp. 137-140 ; Des Garets V. (1999), Études et recherches commerciales, Paris, Economica.
De façon opérationnelle, les variables cognitives collectées dans ces différentes recherches sont assez similaires (Dhar et Nowlis, 1999 ; Dhar, Nowlis et Sherman, 2000 ; Chu et Spires, 2001 ; Weenig et Maarleveld, 2002 ; Petr et Hess-Miglioretti, 2010). Quatre types de données sont nécessaires pour décrire le processus cognitif suivi par le consommateur : la quantité d'informations (le nombre d'informations utilisées), exprimée en valeur absolue et en pourcentage du nombre total ; la nature de l'information (le prix, les caractéristiques techniques, esthétiques...), avec des moyennes calculées sur chaque attribut et le mode d'acquisition des informations, distinguant les stratégies intra-alternatives et intraattributs et la séquence de traitement. En plus de ces variables génériques (présentées au Tableau 3) ont été calculés deux scores permettant de décrire le changement de règle décisionnel : le score de Payne 
Tableau 2. - Présentations des expérimentations assistées par ordinateur sur la pression temporelle

\begin{tabular}{|c|c|c|c|c|}
\hline Choix du produit testé & $\begin{array}{l}\text { Éléments d'information décrivant } \\
\text { l'offre }\end{array}$ & Variables dépendantes & Échantillons & Auteurs \\
\hline Choix d'un appartement & $\begin{array}{l}\text { Choix entre } 5 \text { appartements } \\
\text { Chaque appartement est décrit par } 6 \\
\text { attributs (loyer, bruit, taille, propreté, } \\
\text { sol, parking) } \\
\text { Chacun des attributs a entre } 5 \text { et } 3 \\
\text { valeurs possibles }\end{array}$ & $\begin{array}{l}\text { Temps moyen par } \\
\text { cellule } \\
\text { Nombre de cellules } \\
\text { consultées } \\
\text { Choix effectué }\end{array}$ & $\begin{array}{l}70 \text { étudiants } \\
\text { (répartis en deux } \\
\text { échantillons, un } \\
\text { avec et un sans } \\
\text { PT) }\end{array}$ & $\begin{array}{l}\text { Chu et Spires } \\
(2001)\end{array}$ \\
\hline $\begin{array}{l}\text { Choix d'une télévision, } \\
\text { d'un micro-onde et d'un } \\
\text { appareil photo }\end{array}$ & $\begin{array}{l}\text { Choix entre deux modèles (modèle A et } \\
\text { B) } \\
\text { Chaque produit a } 5 \text { caractéristiques } \\
\text { (pour le téléviseur : la qualité de } \\
\text { l'image, de nombre de hauts parleurs, la } \\
\text { taille de l'écran, les autres fonctions et } \\
\text { le prix) } \\
\text { Chaque attribut a une valeur propre }\end{array}$ & \multirow{2}{*}{$\begin{array}{l}\text { Temps moyen par } \\
\text { cellule } \\
\text { Nombre et ordre des } \\
\text { cellules consultées } \\
\text { Mode de transitions } \\
\text { entre deux cellules (intra- } \\
\text { alternatives ou intra - } \\
\text { attributs ) } \\
\text { Achat ou report de la } \\
\text { décision }\end{array}$} & $\begin{array}{l}143 \text { étudiants } \\
\text { (répartis en deux } \\
\text { échantillons, avec } \\
\text { et sans PT) }\end{array}$ & $\begin{array}{l}\text { Dhar et Nowlis } \\
\text { (1999) }\end{array}$ \\
\hline $\begin{array}{l}\text { Choix de jumelles, d'un } \\
\text { barbecue, d'un PC et } \\
\text { d'une calculatrice }\end{array}$ & $\begin{array}{l}\text { Choix entre trois modèles ( } \mathrm{A}, \mathrm{B} \text { et } \mathrm{C} \text { ) } \\
\text { Chaque modèle a } 4 \text { caractéristiques } \\
\text { Chaque attribut a une valeur propre }\end{array}$ & & $\begin{array}{l}139 \text { étudiants } \\
\text { (deux échantillons, } \\
\text { avec et sans PT) }\end{array}$ & $\begin{array}{l}\text { Dhar et alii } \\
(2000)\end{array}$ \\
\hline Choix d'un réfrigérateur & $\begin{array}{l}\text { Choix entre } 6 \text { modèles } \\
\text { Chaque modèle est décrit par } 6 \text { attributs } \\
\text { (le prix, la durabilité, le volume, la } \\
\text { consommation énergétique, la taille du } \\
\text { congélateur, la capacité de congélation) } \\
\text { Les } 36 \text { informations ont des valeurs } \\
\text { différentes }\end{array}$ & $\begin{array}{l}\text { Identiques aux travaux } \\
\text { de Dhar et alii }(2000) \\
+\end{array}$ & $\begin{array}{l}128 \text { étudiants ( } 2 \\
\text { échantillons, avec } \\
\text { et sans pression } \\
\text { temporelle) }\end{array}$ & $\begin{array}{l}\text { Weenig et } \\
\text { Maarleveld } \\
(2002)\end{array}$ \\
\hline $\begin{array}{l}\text { Choix d'un abonnement } \\
\text { à un fournisseur } \\
\text { d'Internet }\end{array}$ & $\begin{array}{l}\text { Choix entre } 5 \text { modèles } \\
\text { Chaque modèle est décrit par } 7 \text { attributs } \\
\text { (prix du modem et de l'abonnement, } \\
\text { débit, durée de l'engagement, frais de } \\
\text { résiliation, coût téléphonie illimitée et } \\
\text { de la hotline) }\end{array}$ & $\begin{array}{l}\text { Temps moyen par } \\
\text { cellule } \\
\text { Nombre et ordre des } \\
\text { cellules consultées } \\
\text { Mode de transitions } \\
\text { entre deux cellules }\end{array}$ & $\begin{array}{l}904 \text { personnes ( } 2 \\
\text { échantillons, un } \\
\text { par collecte } \\
\text { manuelle et un en } \\
\text { numérique) }\end{array}$ & $\begin{array}{l}\text { Petr et Hess- } \\
\text { Miglioretti } \\
(2010)\end{array}$ \\
\hline
\end{tabular}


(1976) et le score de Transi, spécialement créé pour cette recherche.

Le score de Payne (1976) calcule le degré relatif d'acquisition des informations par attributs par rapport aux stratégies d'acquisition fondées sur les alternatives. Seules les transitions sur un axe orthogonal sont prises en compte. Son mode de calcul est le suivant. Pour chaque participant, le nombre total de transitions intra-attributs est soustrait du nombre total de transitions intra-alternatives, et le tout est divisé par la somme des deux modes de transitions. Sa valeur varie entre -1 et +1 . Ce score est insuffisant pour valider l'hypothèse $\mathrm{H} 5$ qui suppose un lien entre l'intensité de la pression temporelle et le développement de stratégies décisionnelles confuses. En effet, celui-ci ne prend en considération que les transitions effectuées sur les deux axes ; or, le consommateur peut également consulter successivement des informations sans lien direct entre elles. En prenant exemple sur la formule de Payne, le score de Transi (de transitions « aléatoires ») a été créé. Contrairement au premier, celui-ci intègre les transitions « en diagonale ». Une valeur négative traduit une plus forte proportion de transitions en diagonale, et donc plus de stratégies aléatoires que de stratégies fondées sur des comparaisons directes (d'attributs ou d'alternatives). Comme pour le score de Payne, la valeur du score est comprise entre -1 et +1 . Le mode de calcul des deux scores est repris dans le tableau 3.

Concernant la mesure de la pression temporelle, il apparait qu'elle est souvent mesurée par un outil simple, composé d'un seul item sur lequel aucun consensus ne se dégage. Compte tenu du rôle central de cette variable dans le cadre conceptuel développé, cette recherche préconise l'utilisation de trois items complémentaires. L'objectif de l'outil de mesure de la pression temporelle est double : il doit apprécier l'intensité du sentiment et envisager les trois fonctions assignées au concept, considéré comme un antécédent, une manifestation ou une conséquence. À la suite de Weenig et Maarleveld (2002) qui recommandent deux items, le Tableau 5 présente les trois items utilisés, leur mode de mesure ainsi que l'apport visé par chacun des items.

\section{Plan expérimental}

Le plan expérimental de cette recherche repose sur une affectation au hasard du sujet à un des trois groupes : le groupe de contrôle «sans pression temporelle »; le groupe expérimental «pression temporelle modérée » et le groupe expérimental «pression temporelle forte ». Trois scénarios semblables, qui diffèrent seulement dans leurs perspectives temporelles, ont été construits. La situation d'achat est identique et, à l'instar de toutes les recherches expérimentales sur le sujet, la pression temporelle est principalement induite par la présence d'un temps restreint pour le traitement des informations. L'échéance imposée est double, à la fois externe et situationnelle («vous partez dans deux jours »), mais également interne et choisie par le sujet («vous devez vous décider aujourd'hui »). Le calcul du temps disponible de traitement des informations laissé aux sujets est un point délicat. Dhar et ses collègues (Dhar et Nowlis, 1999 ; Dhar, Nowlis et Sherman, 2000) fixent ce temps arbitrairement en fonction du nombre de produits étudiés : 15 secondes par catégorie de produit à examiner. D'autres préfèrent prendre pour base de calcul d'un temps moyen, auprès d'un échantillon de contrôle, qu'ils réduisent de $25 \%, 50 \%$ ou $75 \%$ (Chu et Spires, 2001 ; Weenig et Maarleveld, 2002). C'est cette dernière méthode qui a été appliquée, à la suite d'un pré-test caractérisé par un temps moyen de 135 secondes (écart type : 85 secondes, minimum 13 secondes, maximum 329 secondes). Les trois conditions expérimentales sont présentées dans le Tableau 5.

\section{Opérationnalisation de la méthode}

L'opérationnalisation de la méthode a supposé la construction d'un programme informatique spécifique. Il existe bien, aux États-Unis, des logiciels prêts à l'emploi nommés «Deal Search » (Schurr et Brucks, 1991), mais ceux-ci requièrent des connaissances avancées dans la programmation informatique et sont peu adaptables aux objectifs précis assignés à cette recherche ${ }^{10}$. Le choix s'est alors porté sur la

10. On peut noter qu'il existe, depuis peu, une application Web gratuite proposant des possibilités de réaliser des tables d'information en ligne (Petr et Hess-Miglioretti, 2010). 


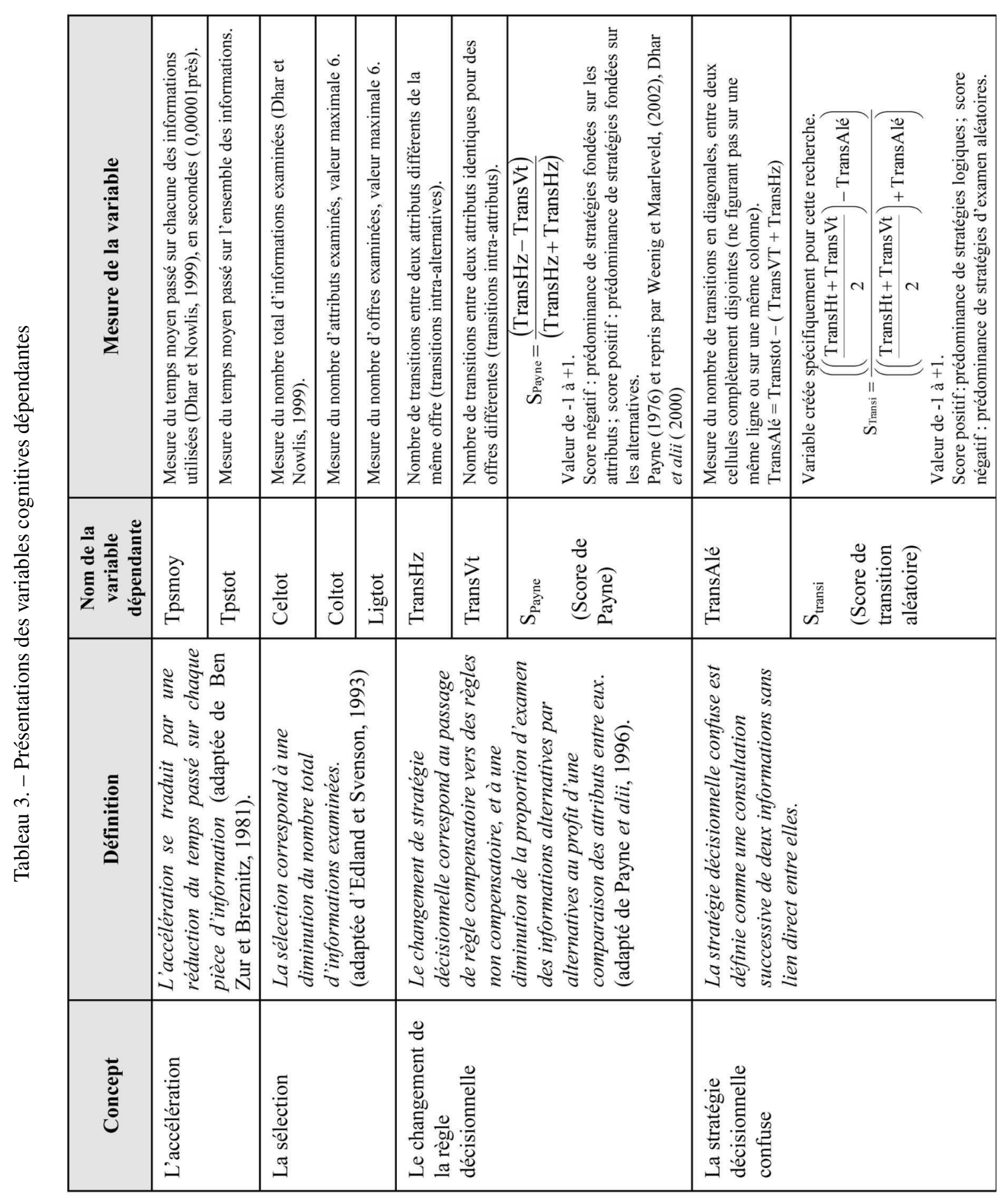


Tableau 4. - Mesure de la pression temporelle

\begin{tabular}{|c|c|c|}
\hline $\begin{array}{c}\text { Dimension de la } \\
\text { pression } \\
\text { temporelle }\end{array}$ & Items et mesure & Auteurs \\
\hline $\begin{array}{l}\text { Item PT1 } \\
\text { Antécédente }\end{array}$ & $\begin{array}{l}\text { Avez-vous eu suffisamment de temps pour faire votre choix? } \\
\text { Mesuré sur une échelle en } 7 \text { points de } \\
\text { Temps pas du tout suffisant à }\end{array}$ & Nowlis (1995) \\
\hline $\begin{array}{c}\text { Item PT2 } \\
\text { Manifestation }\end{array}$ & $\begin{array}{l}\text { Au cours de votre choix, avez-vous eu le sentiment d'être pressé? } \\
\text { Mesuré sur une échelle en } 7 \text { points de } \\
\text { Je n'étais aucunement pressé à 'àtais fortement pressé }\end{array}$ & $\begin{array}{l}\text { Payne et alii (1996) } \\
\text { Weenig et } \\
\text { Maarleveld (2002) }\end{array}$ \\
\hline $\begin{array}{c}\text { Item PT3 } \\
\text { Conséquence }\end{array}$ & $\begin{array}{l}\text { Plus de temps vous aurait-il permis de faire un meilleur choix? } \\
\text { Mesuré sur une échelle en } 7 \text { points de } \\
\text { Pas du tout d'accord }\end{array}$ & $\begin{array}{l}\text { Weenig et } \\
\text { Maarleveld (2002) } \\
\text { Topi et alii (2005) }\end{array}$ \\
\hline
\end{tabular}

Tableau 5. - Présentation du plan expérimental

\begin{tabular}{|c|c|c|c|c|c|c|}
\hline $\begin{array}{c}\text { Présentation des } 3 \\
\text { scénarios }\end{array}$ & $\begin{array}{c}\text { Situation d'achat } \\
\text { proposée }\end{array}$ & \multicolumn{2}{|c|}{$\begin{array}{c}\text { Échéances externes et } \\
\text { internes }\end{array}$} & $\begin{array}{l}\text { Temps disponible } \\
\text { pour le traitement } \\
\text { des informations }\end{array}$ & \multicolumn{2}{|c|}{$\begin{array}{c}\text { Visualisation } \\
\text { du temps } \\
\text { restant }\end{array}$} \\
\hline $\begin{array}{l}\text { Scénario sans } \\
\text { pression } \\
\text { temporelle }\end{array}$ & \multirow{3}{*}{$\begin{array}{l}\text { «Vous allez partir en } \\
\text { déplacement pour trois } \\
\text { mois, vous devez } \\
\text { acheter un ordinateur } \\
\text { avant de partir. » }\end{array}$} & \multicolumn{2}{|c|}{ Pas d'échéance } & $\begin{array}{l}\text { Temps moyen de } \\
\text { traitement des } \\
\text { informations : } \\
135 \text { secondes }(2,25 \\
\text { minutes })\end{array}$ & \multicolumn{2}{|c|}{ Aucune } \\
\hline $\begin{array}{c}\text { Scénario avec une } \\
\text { pression } \\
\text { temporelle } \\
\text { modérée }\end{array}$ & & \multirow{2}{*}{$\begin{array}{l}\frac{\text { Échéance }}{\frac{\text { externe }}{\text { Modéré }}} \\
\text { "Vous } \\
\text { partez dans } \\
\text { deux } \\
\text { jours» }\end{array}$} & \multirow{2}{*}{$\begin{array}{l}\frac{\text { Échéance }}{\text { interne }} \\
\text { Forte } \\
\text { "Vous devez } \\
\text { vous décider } \\
\text { aujourd'hui " }\end{array}$} & $\begin{array}{l}\text { Temps disponible pour le } \\
\text { traitement des } \\
\text { informations : } \\
2 \text { minutes }\end{array}$ & $\begin{array}{l}\text { Sablier } \\
\text { avec } \\
\text { les } \\
\text { secondes } \\
\text { restantes }\end{array}$ & $\begin{array}{c}120 \\
\rightarrow\end{array}$ \\
\hline $\begin{array}{c}\text { Scénario avec une } \\
\text { pression } \\
\text { temporelle forte }\end{array}$ & & & & $\begin{array}{l}\text { Temps disponible pour le } \\
\text { traitement des } \\
\text { informations : } \\
1 \text { minute }\end{array}$ & $\begin{array}{l}\text { Sablier } \\
\text { avec } \\
\text { les } \\
\text { secondes } \\
\text { restantes }\end{array}$ & $\begin{array}{l}60 \\
8\end{array}$ \\
\hline
\end{tabular}

création d'un programme informatique spécifique assistée par une micro-entreprise spécialisée dans les sites Internet. ${ }^{11}$

11. L'auteur tient à remercier le laboratoire de recherche CERMAT de l'Université de Tours qui a financé le programme informatique nécessaire à cette procédure.
La mise en œuvre de la méthode a nécessité plusieurs étapes. Le choix de l'ordinateur portable pour cette recherche se justifie par plusieurs raisons. En premier, un focus group a montré que l'ordinateur portable peut correspondre à un achat en situation de pression temporelle. En second, l'achat d'un ordinateur portable a l'avantage d'être réalisé par des 
hommes et femmes de tout âge et catégories socioprofessionnelles. Troisième raison : la diversité et la vitesse de renouvellement des offres sont telles que les consommateurs doivent obligatoirement se renseigner avant d'acheter. Enfin, les consommateurs sont habitués à une présentation de l'offre sous forme numérique avec un format proche de celui utilisé dans la méthode développée ${ }^{12}$.

Trois pré-tests successifs, réalisés en laboratoires, ont été nécessaires pour tester le programme, valider la procédure et la compréhension des intitulés des attributs, préciser les temps nécessaires et épurer les échelles. Un quatrième pré-test a permis de faire un test en blanc de la procédure et de son envoi par Internet. À la suite de ceux-ci, les choix suivants ont été opérés. Le programme informatique a permis d'affecter à chaque participant un des trois scénarios au hasard. Les places des attributs dans la matrice ont été choisies de façon aléatoire et celles-ci sont restées identiques pour tous les participants. L'adresse Internet hébergeant le site a été envoyée à une centaine de sujets qui, à leur tour, l'ont transférée vers d'autres individus. En trois semaines, 580 réponses ont été collectées. Une relecture des données a abouti à l'élimination de certains questionnaires fantaisistes ou incomplets. Finalement, l'échantillon final comporte 521 répondants, avec des caractéristiques socio-économiques proches des acheteurs d'ordinateur $(45,8 \%$ de femmes ; $44 \%$ de cadres, $35 \%$ d'employés et $35 \%$ d'étudiants). Cette base a permis de réaliser les tests des hypothèses par le biais d'analyses de la variance et de régressions simples et multiples.

\section{RÉSULTATS}

\section{Validation de la procédure expérimentale}

Pour percevoir si les trois situations sont appréhendées comme plus ou moins «pressantes », cinq analyses de la variance ont été réalisées. L'objectif de ces analyses est de vérifier qu'il existe un lien de causalité entre une variable explicative (le temps contraint, variable nominale en trois niveaux) et les cinq variables dépendantes témoins de la réussite de la manipulation, composées des trois variables dépendantes (PT01, PT02 et PT03) qui mesurent en sept points l'intensité de la pression temporelle, du score factoriel (FACTPTS) obtenu par le regroupement de ces trois items et présenté comme un outil de mesure simple, unidimensionnel et fiable de la pression temporelle, et de la variable dépendante métrique (Tpstot) qui rapporte le temps total passé à l'examen de la matrice. Les analyses de variance confirment que les deux scénarios avec pression (modérée et forte) induisent un sentiment de pression temporelle plus fort que le scénario sans contrainte de temps. Les valeurs du test F sont significatives (Tableau 6). Comme attendu, les consommateurs dans les deux situations de pression temporelle expriment un plus fort sentiment de pression temporelle que ceux qui sont sans contrainte. En moyenne, les consommateurs sans pression temporelle utilisent trois fois plus de temps (168 secondes en moyenne) que ceux qui sont dans une situation de temps fortement contraint (54 secondes). En outre, la situation «pression temporelle forte » engendre un sentiment de plus forte intensité que celle avec une «pression temporelle modérée ».

\section{Validation de l'accélération avec un effet de seuil}

L'intensité de la pression temporelle a des effets complexes sur le temps moyen passé sur chaque information. Un premier examen des statistiques descriptives semble valider le principe d'accélération décrit dans les recherches antérieures (Tableau 7). Ainsi, la mise en commun des deux plans expérimentaux, permettant de créer un variable binaire - avec
12. En 2008, $78 \%$ des consommateurs interrogés ont consulté Internet avant d'effectuer un achat, avec une préférence pour les guides d'achat des distributeurs, des sites spécialisés, et des avis d'internautes (Étude de la Fédération du $e$-commerce et de la vente à distance). 
Tableau 6. - Analyse de la variance testant l'effet de la manipulation expérimentale sur le sentiment de pression temporelle

\begin{tabular}{|c|c|c|c|c|c|c|c|c|c|}
\hline & \multicolumn{2}{|c|}{ Item PT1 } & \multicolumn{2}{|c|}{ Item PT2 } & \multicolumn{2}{|c|}{ Item PT3 } & \multicolumn{2}{|c|}{$\begin{array}{l}\text { Temps total consacré à } \\
\text { la recherche } \\
\text { (en secondes) }\end{array}$} & \multirow{2}{*}{$\begin{array}{c}\text { Score factoriel } \\
\text { FactPTS }\end{array}$} \\
\hline & Moyenne & $\begin{array}{l}\text { Écart } \\
\text { type }\end{array}$ & Moyenne & $\begin{array}{l}\text { Écart } \\
\text { type }\end{array}$ & Moyenne & $\begin{array}{l}\text { Écart } \\
\text { type }\end{array}$ & Moyenne & Écart type & \\
\hline $\begin{array}{l}\text { Sans pression } \\
\text { temporelle }\end{array}$ & 3,22 & 1,9 & 3,56 & 2,02 & 3,55 & 2,08 & 168,67 & 154,24 & \multirow{4}{*}{$\begin{array}{c}F=109,681 \\
p<0,001\end{array}$} \\
\hline $\begin{array}{l}\text { Avec pression } \\
\text { temporelle modérée }\end{array}$ & 5,57 & 1,8 & 5,67 & 1,75 & 5,13 & 1,90 & 75,01 & 27,65 & \\
\hline $\begin{array}{l}\text { Avec pression } \\
\text { temporelle forte }\end{array}$ & 5,99 & 1,6 & 5,97 & 1,78 & 5,68 & 1,84 & 54,95 & 9,79 & \\
\hline Anova & \multicolumn{2}{|c|}{$F=107,28 ; p<0,01$} & \multicolumn{2}{|c|}{$\begin{array}{c}\mathrm{F}=77,12 ; \mathrm{p}< \\
0,001\end{array}$} & \multicolumn{2}{|c|}{$\mathrm{F}=49,96 ; \mathrm{p}<0,001$} & \multicolumn{2}{|c|}{$F=85,105 ; p<0,001$} & \\
\hline
\end{tabular}

ou sans pression temporelle - indique que le temps moyen passé sur chaque information est réduit en situation de temps contraint (de 6,8 à 5,3 secondes). Pourtant, il apparaît que le temps moyen passé sur chaque élément d'information est plus long pour une pression temporelle forte $(5,9$ secondes $)$ que pour une pression temporelle modérée ( 4,7 secondes) !

Une comparaison des moyennes a priori permet de préciser l'effet de l'intensité de la pression temporelle sur l'accélération. En appliquant des coefficients de contrastes permettant de comparer deux à deux chaque situation (Tableau 8), on constate que le principe d'accélération est vérifié globalement, dans le cas où la comparaison s'effectue entre un scénario
« avec » et « sans pression temporelle » et que la différence entre la pression modérée et la pression forte est également significative. Lorsque la pression temporelle s'intensifie, le temps moyen croît. En revanche, il n'y a pas de différence significative du temps moyen passé sur chaque information entre une expérience menée avec un temps non contraint et un temps fortement contraint. H1 est vérifiée. Si ces résultats confirment, en cohérence avec les travaux passés, le principe d'accélération, ils démontrent également, de façon inédite, que l'accélération n'est pas un processus linéaire. La pression temporelle décrit sur l'accélération une courbe avec un effet de seuil lié à son intensité.

Tableau 7. - Moyennes et écarts types du temps moyen passé sur chaque information en fonction du scénario proposé

\begin{tabular}{|c|c|c|c|c|}
\hline $\begin{array}{c}\text { Temps passé sur } \\
\text { chaque information } \\
\text { Variable dépendante } \\
\text { (Tpsmoy) }\end{array}$ & $\begin{array}{c}\text { Sans pression } \\
\text { temporelle }\end{array}$ & $\begin{array}{c}\text { Avec pression } \\
\text { temporelle } \\
\text { (modérée et forte) }\end{array}$ & $\begin{array}{c}\text { Avec pression } \\
\text { temporelle modérée }\end{array}$ & $\begin{array}{c}\text { Avec pression } \\
\text { temporelle forte }\end{array}$ \\
\hline Moyenne (secondes) & 6,8422 & 5,326 & 4,749 & 5,925 \\
\hline Ecart type & 6,651 & 3,991 & 2,886 & 4,817 \\
\hline $\mathrm{N}=521$ & 138 & 383 & 195 & 188 \\
\hline
\end{tabular}


Tableau 8. - Test des contrastes sur le temps moyen passé sur chaque information

\begin{tabular}{|c|c|c|c|c|c|c|}
\hline \multirow{3}{*}{$\begin{array}{c}\text { Temps } \\
\text { moyen passé } \\
\text { sur chaque } \\
\text { information }\end{array}$} & Contrastes & $\begin{array}{l}\text { Valeur du } \\
\text { contraste }\end{array}$ & Écart type & T de Student & $\begin{array}{l}\text { Degrés de } \\
\text { liberté }\end{array}$ & Significativité \\
\hline & $\begin{array}{c}\text { Entre PT modérée } \\
\text { et } P T \text { forte }\end{array}$ & $-1,257$ & 0,488 & $-2,574$ & 517 & 0,010 \\
\hline & $\begin{array}{c}\text { Entre sans } P T \text { et } \\
P T\end{array}$ & 1,545 & 0,474 & 3,261 & 517 & 0,001 \\
\hline (Tpsmoy) & $\begin{array}{c}\text { Entre sans } P T \text { et } \\
P T \text { forte }\end{array}$ & 0,916 & 0,534 & 1,715 & 517 & 0,087 \\
\hline
\end{tabular}

\section{Validation d'un processus sélectif linéaire}

L'hypothèse $\mathrm{H} 2$ propose de vérifier l'effet de l'intensité de la pression temporelle sur le nombre d'informations consultées (H2a), le nombre d'attributs pris en compte $(\mathrm{H} 2 \mathrm{~b})$ et le nombre d'alternatives consultées (H2c). Les statistiques descriptives indiquent que le nombre d'éléments pris en compte baisse de façon significative et que le principe de sélection est un mécanisme qui touche plus sévèrement les attributs que les marques (Tableau 9). Globalement les sujets, dans un contexte de pression temporelle forte, consultent $40 \%$ d'informations en moins par comparaison avec ceux qui subissent une faible pression, et l'écart est de $60 \%$ avec ceux qui sont sans contrainte temporelle. L'analyse de la variance, complétée du test des contrastes a priori, révèle une différence des moyennes significative entre les trois scénarios. H2a est validée, plus le temps du consommateur pour faire son choix est contraint, et plus sa sélection est globalement importante. De la même façon, les analyses de variances testant l'effet de la pression temporelle sur la baisse du nombre de marques examinées (5,6 sans PT, 5,5 avec PT modérée, 5,1 avec PT forte) et d'attributs (4,9 sans PT, 4,2 avec PT modérée, 3,5 avec PT forte) sont significatives. En condition de forte pression, l'ensemble de considération des consommateurs est réduit d'une marque et de trois des six attributs. Les tests a priori effectués par la méthode des contrastes indiquent que le sujet sélectionne de façon graduelle selon l'intensité de la pression temporelle les marques et les attributs qu'il prend en considération. Les hypothèses H2b et H2c sont validées.

Tableau 9. - Analyse de la variance testant l'effet de la pression temporelle sur la sélection

\begin{tabular}{|c|c|c|c|c|c|c|c|c|c|c|c|c|}
\hline \multirow{2}{*}{ Variable dépendante } & \multicolumn{3}{|c|}{ Moyenne } & \multicolumn{2}{|c|}{ Test de Levene } & \multicolumn{2}{|c|}{ Test de Welsh } & \multicolumn{5}{|c|}{ Anova } \\
\hline & $\begin{array}{c}\text { Sans } \\
P T\end{array}$ & $\begin{array}{c}P T \\
\text { mod. }\end{array}$ & $\begin{array}{c}P T \\
\text { forte }\end{array}$ & $\mathrm{F}$ & $\mathrm{p}$ & $\mathrm{F}$ & $\mathrm{p}$ & ddl & $\mathrm{F}$ & $\mathrm{p}$ & Eta & $\mathrm{Eta}^{2}$ \\
\hline $\begin{array}{c}\text { Nombre } \\
\text { d'informations } \\
\text { examinées } \\
\text { (Celtot) }\end{array}$ & 28,26 & 18,16 & 12,36 & 1,359 & 0,258 & 53,542 & 0,000 & 512 & 52,611 & 0,000 & 0,21 & 0,04 \\
\hline $\begin{array}{l}\text { Nombre d'offres } \\
\text { examinées } \\
\text { (Ligtot) }\end{array}$ & 5,67 & 5,51 & 5,11 & 18,77 & 0,000 & 9,44 & 0,000 & 512 & 7,11 & 0,001 & 0,03 & 0,009 \\
\hline $\begin{array}{c}\text { Nombre } \\
\text { d'attributs } \\
\text { examinés } \\
\text { (Coltot) }\end{array}$ & 4,90 & 4,2 & 3,5 & 7,06 & 0,001 & 31,99 & 0,000 & 512 & 30,22 & 0,000 & 0,12 & 0,014 \\
\hline
\end{tabular}


En suivant le protocole proposé par Weenig et Maarleveld (2002), l'hypothèse H3 est testée à l'aide du ratio de sélectivité, obtenu en rapportant le nombre de fréquentations de la colonne en situation de pression temporelle au nombre de fréquentations sans contrainte temporelle. Une comparaison des moyennes a été ensuite effectuée entre les deux échantillons indépendants (avec et sans pression) à l'aide d'un test $t$ de Student. Le Tableau 10 montre que la consultation des informations baisse significativement pour tous les attributs proposés, les deux attributs les moins affectés par la pression temporelle sont les attributs considérés comme les plus importants : la marque et le prix (leur baisse de consultation respective n'est que de $30 \%$ et $40 \%$ ) ; la plus forte diminution de fréquentation de la colonne concerne les attributs considérés comme les moins importants : les modèles, leurs photos et les coloris disponibles (la baisse est de $60 \%$ ). L'hypothèse $\mathbf{H 3}$ est validée. La sélection est un processus linéaire par rapport à l'importance relative accordée aux informations.

\section{Principe de changement de la règle décisionnelle}

Contrairement à ce qui était supposé, le score de Payne reste au même niveau (proche de -0,4) et négatif, quelle que soit l'intensité de la pression temporelle. Ceci signifie que la stratégie choisie par les individus est majoritairement intra-attributs, indépendamment de l'intensité de la pression. Les sujets examinent beaucoup plus les informations deux à deux sur la base des mêmes caractéristiques que sur des alternatives complètes. Les résultats de l'analyse de la variance confirment cet effet non significatif (Tableau 11). L'hypothèse H4 n'est pas validée. Quelle que soit l'intensité de la pression, le consommateur préfère une stratégie décisionnelle de type intra-attributs à une stratégie décisionnelle intra-alternative.

L'hypothèse H5 a pour objectif de tester la compensation entre les mécanismes d'accélération et la sélection. Compte tenu de la nature de ces deux variables, il a d'abord été procédé à une analyse des corrélations. La matrice fournit une première indication de la relation entre l'accélération et la sélection, puisque la corrélation est significative, de signe négatif avec $r=-0,452(p<0,01)$. Cette corrélation étant inférieure au seuil généralement admis de 0,70 , il a été procédé à une analyse de régression entre le temps moyen passé sur chaque pièce d'information et le nombre de cellules consultées. Les indicateurs (Tableau 12) confirment l'existence d'un processus complémentaire. Le coefficient de régression linéaire significatif est négatif, ce qui traduit que plus le temps moyen par élément d'information est long, plus la sélection est importante. En d'autres termes, l'accélération et la sélection sont en sens opposé, ce

Tableau 10. - Ratios de sélectivité sur les différents attributs

\begin{tabular}{|c|c|c|c|c|c|c|c|c|c|}
\hline & \multicolumn{3}{|c|}{$\begin{array}{c}\text { Nombre d'informations } \\
\text { consultées sans PT }\end{array}$} & \multicolumn{2}{c|}{$\begin{array}{c}\text { Nombre d'informations } \\
\text { consultées avec PT } \\
\mathrm{M}_{\mathrm{pt}}\end{array}$} & \multicolumn{4}{c|}{$\begin{array}{c}\text { Ratio de sélectivité } \\
\mathbf{M}_{\mathrm{pt}} / \mathbf{M}_{\mathrm{spt}}\end{array}$} \\
\hline & Rang & Moyenne & $\begin{array}{c}\text { Écart } \\
\text { type }\end{array}$ & Rang & Moyenne & $\begin{array}{c}\text { Écart } \\
\text { type }\end{array}$ & Rang & Ratio & $p$ \\
\hline Prix & 1 & 6,62 & 5,7 & 1 & 3,90 & 3,69 & 3 & 0,59 & $<0,001$ \\
\hline Marque & 2 & 4,95 & 2,5 & 2 & 3,40 & 2,85 & 1 & 0,69 & $<0,001$ \\
\hline Info conso & 3 & 4,90 & 4,18 & 3 & 2,98 & 3,29 & 2 & 0,61 & $<0,001$ \\
\hline Capacité & 5 & 4,52 & 6,29 & 4 & 2,42 & 3,04 & 4 & 0,54 & $<0,001$ \\
\hline Utilisation & 4 & 4,63 & 6,37 & 5 & 1,47 & 2,18 & 6 & 0,32 & $<0,001$ \\
\hline Modèle & 6 & 2,63 & 3,33 & 6 & 1,03 & 1,90 & 5 & 0,39 & $<0,001$ \\
\hline
\end{tabular}


Tableau 11. - Analyse de la variance testant l'effet de la pression temporelle sur le score de Payne

\begin{tabular}{|c|c|c|c|c|c|c|c|c|c|c|c|c|}
\hline \multirow{2}{*}{$\begin{array}{c}\text { Variable } \\
\text { dépendante }\end{array}$} & \multicolumn{3}{|c|}{ Moyenne } & \multicolumn{2}{|c|}{ Test de Levene } & \multicolumn{2}{|c|}{ Test de Welsh } & \multicolumn{5}{|c|}{ Anova } \\
\hline & $\begin{array}{c}\text { Sans } \\
P T\end{array}$ & $\begin{array}{l}P T \\
\bmod \end{array}$ & PT forte & $\mathrm{F}$ & $\mathrm{p}$ & $\mathrm{F}$ & $\mathrm{p}$ & ddl & $\mathrm{F}$ & $\mathrm{p}$ & Eta & $\mathrm{Eta}^{2}$ \\
\hline $\begin{array}{l}\text { Score de } \\
\text { Payne }\end{array}$ & $-0,415$ & $-0,443$ & $-0,432$ & 2,030 & 0,132 & 0,219 & 0,640 & 520 & 0,122 & 0,886 & 0,00 & 0,000 \\
\hline
\end{tabular}

Tableau 12. - Régression simple entre l'accélération et la sélection

\begin{tabular}{|c|c|c|c|c|c|c|c|}
\hline $\begin{array}{c}\text { Variable } \\
\text { dépendante }\end{array}$ & F & ddl & $\mathbf{P}$ & $\mathbf{R}^{2}$ ajusté & Bêta & $\mathbf{t}$ & $\mathbf{p}$ \\
\hline $\begin{array}{c}\text { Nombre } \\
\text { d'informations } \\
\text { examinées } \\
\text { (Celtot) }\end{array}$ & 427,296 & 377 & 0,000 & 0,531 & $-0,729$ & $-20,6711$ & 0,000 \\
\hline \\
Variable indépendante, Tpsmoy (temps moyen); PT =1, variable fixée, avec un scénario de pression temporelle \\
\hline
\end{tabular}

sont des mécanismes qui se compensent. L'hypothèse $\mathrm{H5}$ est validée. En situation de temps contraint, l'accélération et la sélection sont des méthodes exclusives plutôt qu'additives. Cette régression explique $53 \%$ de la variance. En revanche, sans contrainte temporelle, il n'y a pas de relation entre le nombre d'informations consultées et la durée passée sur chacune d'entre elles.

L'hypothèse H6 suppose que plus la pression temporelle est forte, plus la stratégie du consommateur est confuse. Sur ce score de Transi, pour lequel il n'existe pas de comparaisons dans les travaux antérieurs, les moyennes sont positives et proches de 0,5 , ce qui indique que les transitions orthogonales sont trois fois plus importantes que les transitions diagonales. Autrement dit, les consommateurs procèdent généralement de façon « logique et rationnelle », en comparant deux à deux des informations proches. Cependant, la diminution de la moyenne est significative, comme le confirment les résultats de l'analyse de la variance (Tableau 13). Le score est de 0,66 sans contrainte de temps contre 0,44 en situation de forte pression temporelle. Plus le temps est contraint, moins le processus adopté par le consommateur est orthogonal : l'hypothèse $\mathbf{H 6}$ est validée. Si la pression temporelle suscite un mode d'examen moins logique que dans un contexte sans temps contraint, ces résultats réfutent le principe de stratégie d'examen confuse. Celle-ci reste rigoureuse, même en situation de forte pression temporelle.

Tableau 13. - Analyse de la variance testant l'effet de la pression temporelle sur le score de Transi

\begin{tabular}{|c|c|c|c|c|c|c|c|c|c|c|c|c|}
\hline \multirow{2}{*}{$\begin{array}{l}\text { Variable } \\
\text { dépendante }\end{array}$} & \multicolumn{3}{|c|}{ Moyenne } & \multicolumn{2}{|c|}{ Test de Levene } & \multicolumn{2}{|c|}{ Test de Welsh } & \multicolumn{5}{|c|}{ Anova } \\
\hline & $\begin{array}{c}\text { Sans } \\
P T\end{array}$ & PT mod & PT forte & F & $\mathrm{p}$ & $\mathrm{F}$ & $\mathrm{p}$ & ddl & $\mathrm{F}$ & $\mathrm{p}$ & Eta & $\mathrm{Eta}^{2}$ \\
\hline $\begin{array}{c}\text { Score de } \\
\text { Transitions } \\
\text { « aléatoires » } \\
\text { (Transi) }\end{array}$ & 0,663 & 0,470 & 0,445 & 4,304 & 0,014 & 17,823 & 0,000 & 518 & 11,555 & 0,000 & 0,04 & 0,0002 \\
\hline
\end{tabular}


APPORTS, IMPLICATIONS MANAGÉRIALES, LIMITES

\section{Apports}

Les premiers résultats de cette recherche vérifient, sur un échantillon et dans un contexte francophone, des résultats issus de travaux antérieurs. Ainsi, il apparaît que le consommateur sélectionne les informations qu'il souhaite traiter. On retrouve ces résultats dans les travaux de Beach (1993) qui a montré que, face à des choix complexes, les consommateurs s'adaptent en se «cachant » les informations qui ne respectent pas un seuil minimal d'acceptation. Les auteurs (Benson et Beach, 1996) décrivent une théorie, appelée "théorie des images », considérant la façon dont les sujets font un premier examen visuel des contenus. Selon celle-ci, les consommateurs testent la compatibilité d'une option avec leurs attentes par un système de "passage au crible ». Cette expression désigne un processus mental, adopté par les sujets, où les décisions, normalement non dichotomiques, sont réduites à un processus de rejet/acceptation, nécessitant moins d'efforts. Le tableau d'information est «passé au crible », et le choix est «dichotomisé », simplifié. Ce processus de sélection est logique par rapport aux priorités du sujet. En situation de contrainte temporelle, le consommateur passe de 6 marques considérées à 5 , et ne garde que 3 ou 4 attributs essentiels en ignorant ceux qui sont inférieurs au seuil d'acceptation. Les caractéristiques mineures de l'offre sont les premières ignorées. En outre, le consommateur s'adapte à la pression par l'accélération OU la sélection; les deux mécanismes sont substituables. Deux explications peuvent être proposées. La première repose sur le style cognitif des individus. Ceux-ci manifestent une préférence stable pour un mode ou l'autre, en fonction de leurs caractéristiques individuelles. La seconde réside dans l'intensité de la pression temporelle. Lorsque l'accélération devient un processus d'adaptation insuffisant face à une contrainte temporelle plus forte, la sélection est une réponse plus adaptée.

Parallèlement, des nouveaux apports sont à souligner. Ainsi, si le principe d'accélération s'observe de façon générale en situation de pression temporelle par rapport à une situation sans pression, un examen plus minutieux révèle l'existence d'un seuil, audelà duquel s'observe un phénomène de décélération. Le processus d'examen des informations est plus long lorsque la pression temporelle est forte que lorsque celle-ci est modérée. On peut supposer, dans une optique de rationalisation du temps disponible, que le sujet accorde une plus grande attention à chaque élément d'information. La décélération serait-elle un mode cognitif d'adaptation plus qualitatif en situation de choix complexe ? En outre, l'examen des informations observées dans cette recherche est toujours de type intra-attributs, et le reste, quelles que soient les modifications de la manipulation. La pression temporelle n'entraîne pas de changement de la règle décisionnelle. Cette stabilité du processus peut être expliquée par la nature de la tâche. Lorsque celle-ci est complexe, l'examen comparatif des attributs est plus adapté. Finalement, la méthode de recherche d'information est logique, et ne se dégrade que légèrement lorsque la pression s'intensifie. Contrairement à ce qu' avançaient les travaux de Chien-Huang et Pei-Hsun (2005), le consommateur n'adopte pas une «stratégie décisionnelle confuse ». La décélération, la sélection stricte et linéaire des attributs selon l'intensité de la pression, la règle décisionnelle adoptée sont autant de preuves d'une stratégie décisionnelle rigoureuse et raisonnée. Les deux tableaux suivants synthétisent les confirmations et les apports de cette recherche sur les mécanismes d'accélération et de sélection (Tableau 14) ainsi que sur les changements de stratégie décisionnelle (Tableau 15).

Sur un plan méthodologique, ces résultats rappellent la nécessité de manipuler précisément la variable temporelle. Seul un plan d'expérience précis, comportant différents degrés d'intensité de pression, peut permettre d'observer des résultats contrastés (Suri et Monroe, 2003). L'utilisation de deux intensités de contrainte temporelle souligne l'existence de relations curvilignes. Là où les travaux précédents proposaient de différencier les comportements « avec » et " sans pression temporelle », les résultats de cette recherche ajoutent des relations liées à l'intensité de la pression temporelle. L'état de l'art a montré le manque de consensus de nombreux points théoriques précis. Il semblerait logique de croire que ces résultats contradictoires passés s'expliquent en partie par la manipulation expérimentale d'un seul degré de pres- 
Tableau 14. - Synthèse des hypothèses sur la sélection et l'accélération

\begin{tabular}{|c|c|c|c|}
\hline \multirow{2}{*}{\multicolumn{2}{|c|}{ Hypothèses sur l'accélération et la sélection }} & \multicolumn{2}{|c|}{ Synthèse des apports } \\
\hline & & $\begin{array}{l}\text { Confirmations de résultats déjà } \\
\text { testés dans la littérature existante }\end{array}$ & $\begin{array}{c}\text { Nouveaux apports en comparaison } \\
\text { de la littérature existante }\end{array}$ \\
\hline \multirow[b]{2}{*}{ H1 } & \multirow[b]{2}{*}{$\begin{array}{l}\text { ACCÉLÉRATION } \\
\text { Les consommateurs passent plus de temps sur } \\
\text { chaque élément d'information lorsque la pression } \\
\text { temporelle est modérée que lorsque celle-ci est nulle } \\
\text { ou forte. }\end{array}$} & \multicolumn{2}{|c|}{ Hypothèse validée } \\
\hline & & $\begin{array}{c}\text { Validation du principe } \\
\text { d'accélération : } \\
\text { En situation de pression temporelle } \\
\text { par rapport à une situation sans } \\
\text { contrainte de temps, le temps } \\
\text { moyen passé sur chaque élément } \\
\text { est réduit. }\end{array}$ & $\begin{array}{l}\text { Existence d'un effet de } \\
\text { ralentissement en situation de } \\
\text { forte pression temporelle : } \\
\text { L'intensité de la pression } \\
\text { temporelle sur l'accélération décrit } \\
\text { une courbe curviligne, avec un } \\
\text { effet de seuil. }\end{array}$ \\
\hline \multirow[b]{2}{*}{$\begin{array}{l}\mathrm{H} 2 \\
\text { abc }\end{array}$} & \multirow[b]{2}{*}{$\begin{array}{l}\text { SÉLECTION } \\
\text { Plus la pression temporelle est forte, } \\
\text { - plus le nombre global d'informations examinées } \\
\text { diminue. } \\
\text { - plus le nombre d'attributs examinés par le } \\
\text { consommateur diminue. } \\
\text { - plus le nombre d'alternatives examinées par le } \\
\text { consommateur diminue. }\end{array}$} & \multicolumn{2}{|c|}{ Hypothèse validée } \\
\hline & & $\begin{array}{l}\text { Validation du principe de } \\
\text { sélection : } \\
\text { En situation de pression } \\
\text { temporelle, le nombre } \\
\text { d'informations consultées diminue. } \\
\text { La sélection est plus forte sur les } \\
\text { attributs que sur les marques. }\end{array}$ & $\begin{array}{l}\text { Le mécanisme de sélection est } \\
\text { graduel : } \\
\text { Plus son intensité est forte, et plus } \\
\text { la sélection du nombre } \\
\text { d'informations, d'attributs et } \\
\text { d'alternatives est forte. }\end{array}$ \\
\hline \multirow[b]{2}{*}{ H3 } & \multirow[b]{2}{*}{$\begin{array}{l}\text { MODE DE SÉLECTION } \\
\text { Plus la pression temporelle est forte, } \\
\text { plus le processus de sélection s'exerce sur les } \\
\text { attributs considérés d'importance faible comparés } \\
\text { aux attributs de moyenne et de forte importance. }\end{array}$} & \multicolumn{2}{|c|}{ Hypothèse validée } \\
\hline & & $\begin{array}{l}\text { Validation d'un principe de } \\
\text { sélection linéaire par rapport à } \\
\text { l'importance relative accordée } \\
\text { aux informations : } \\
\text { La consultation des caractéristiques } \\
\text { considérées comme primordiales } \\
\text { baisse moins que celle des } \\
\text { informations non essentielles. }\end{array}$ & $\begin{array}{l}\text { La sélectivité ne se fait pas plus } \\
\text { aux dépens des attributs } \\
\text { d'importance modérée que ceux } \\
\text { de faible importance : } \\
\text { Le consommateur garde un } \\
\text { processus rigoureux et raisonné, } \\
\text { même en situation de pression } \\
\text { temporelle forte. }\end{array}$ \\
\hline
\end{tabular}

sion temporelle et la non-maîtrise de l'intensité de celle-ci.

\section{Implications managériales}

Les occasions suscitant une expérience de pression temporelle situationnelle sont nombreuses. De multiples échéances sociétales, les pannes des appareils électroménagers, les offres attractives et éphémères des distributeurs en ligne font qu'un grand nombre d'achats qui devraient «normalement» relever d'un processus extensif, long et rigoureux, sont réalisés sous une contrainte de temps impérative. Les résultats de cette recherche suggèrent deux types d'implications managériales, pour le commerce de détail et le $e$-commerce. Dans un premier temps, il parait judicieux de conseiller aux responsables de magasin de puiser dans les innovations du commerce électronique pour répondre aux exigences d'accélération et de sélection des consommateurs. Trois suggestions peuvent être plus particulièrement formulées : mettre en avant de façon explicite, au sein de l'offre complète, une sélection de produits fondamentaux pour aider la sélection par les consommateurs ; proposer une signalétique catégorisant les offres selon les attributs communs, et ceci afin de favoriser l'accélération par le processus de « passage au crible »; développer dans les points de vente des outils comparatifs à l'image des comparateurs disponibles sur la plupart des sites Internet. Concernant le commerce électronique, les recommandations sont différentes car ceux-ci ont pris conscience, depuis longtemps, du rapport au temps exacerbé des internautes. Les sites de déstockage, comme vente-privee.com, mais aussi les 
Tableau 15. - Synthèse des hypothèses sur le changement de la stratégie décisionnelle

\begin{tabular}{|c|c|c|c|}
\hline \multirow{2}{*}{\multicolumn{2}{|c|}{$\begin{array}{l}\text { Hypothèses sur le changement de stratégie } \\
\text { décisionnelle }\end{array}$}} & \multicolumn{2}{|c|}{ Synthèse des apports } \\
\hline & & $\begin{array}{l}\text { Confirmations de résultats déjà } \\
\text { testés dans la littérature existante }\end{array}$ & $\begin{array}{c}\text { Nouveaux apports en comparaison } \\
\text { de la littérature existante }\end{array}$ \\
\hline \multirow[b]{2}{*}{ H4 } & \multirow[b]{2}{*}{$\begin{array}{l}\text { STRATÉGIE INTRA-ALTERNATIVES ET } \\
\text { STRATÉGIE INTRA-ATTRIBUTS } \\
\text { Plus la pression temporelle est forte, plus la } \\
\text { proportion de stratégies fondées sur des } \\
\text { comparaisons d'attributs augmente, plus la } \\
\text { proportion de stratégies fondées sur des } \\
\text { comparaisons des offres complètes baisse. }\end{array}$} & \multicolumn{2}{|c|}{ Hypothèse non validée } \\
\hline & & $\begin{array}{l}\text { Prédominance des stratégies } \\
\text { intra-attributs en situation de } \\
\text { pression temporelle : } \\
\text { La pression temporelle encourage } \\
\text { un processus comparatif par attribut } \\
\text { plutôt qu'un traitement par } \\
\text { alternative. }\end{array}$ & $\begin{array}{l}\text { Stabilité du mode d'examen des } \\
\text { informations en situation de } \\
\text { pression temporelle : } \\
\text { Le mode de traitement par attribut } \\
\text { reste stable, quelle que soit } \\
\text { l'intensité de la pression } \\
\text { temporelle. }\end{array}$ \\
\hline \multirow[b]{2}{*}{ H5 } & \multirow[b]{2}{*}{$\begin{array}{l}\text { ACCÉLÉRATION ET SÉLECTION } \\
\text { Dans un contexte de pression temporelle, une forte } \\
\text { accélération est associée à une plus faible sélection, } \\
\text { et inversement, une faible accélération correspond à } \\
\text { une forte sélection. }\end{array}$} & \multicolumn{2}{|c|}{ Hypothèse validée } \\
\hline & & $\begin{array}{l}\text { Accélération et sélection sont deux } \\
\text { mécanismes compensatoires : } \\
\text { Le sujet s'adapte à la contrainte } \\
\text { temporelle soit en accélérant son } \\
\text { rythme d'examen des informations, } \\
\text { soit en adoptant un mode plus } \\
\text { sélectif. }\end{array}$ & $\begin{array}{c}\text { En situation de pression } \\
\text { temporelle, l'accélération et la } \\
\text { sélection sont des méthodes } \\
\text { exclusives plutôt qu'additives. } \\
\text { En situation de temps non } \\
\text { contraint, il n'y a pas de relation } \\
\text { entre le nombre d'informations } \\
\text { consultées et la durée passée sur } \\
\text { chacune d'entre elles. }\end{array}$ \\
\hline \multirow[b]{2}{*}{ H6 } & \multirow[b]{2}{*}{$\begin{array}{l}\text { STRATÉGIE ALÉATOIRE } \\
\text { Plus la pression temporelle est forte, } \\
\text { plus le nombre de stratégies décisionnelles aléatoires, } \\
\text { fondées sur des comparaisons d'informations deux à } \\
\text { deux sans lien entre elles, augmente. }\end{array}$} & \multicolumn{2}{|c|}{ Hypothèse non validée } \\
\hline & & $\begin{array}{l}\text { La pression temporelle suscite un } \\
\text { mode d'examen des informations } \\
\text { moins logique que dans un contexte } \\
\text { sans temps contraint. }\end{array}$ & $\begin{array}{l}\text { Réfutation du principe de } \\
\text { stratégie confuse : } \\
\text { En situation de temps contraint, } \\
\text { plus le sentiment de pression } \\
\text { temporelle est intense et plus le } \\
\text { mode adopté est rigoureux et } \\
\text { logique. }\end{array}$ \\
\hline
\end{tabular}

$e$-distributeurs comme la fnac.com ou rue-du-commerce.com ont compris l'intérêt qu'ils avaient à «presser le consommateur ». Si l'effet de la limitation de la durée de l'offre est bénéfique sur l'incitation à l'achat, cette recherche suggère l'existence d'un effet non linéaire lié à l'intensité de la pression temporelle. La pression temporelle modérée n'a pas les mêmes effets qu'une pression temporelle forte, et il est souhaitable que les marques testent précisément les durées à la disposition des internautes avant de s'engager sur cette voie de la contrainte de temps.

\section{Limites}

Cette version informatisée de la méthode des tables d'information offre de nombreux avantages, dont les principaux pour notre questionnement résident dans le maniement très précis de la variable temporelle et la présence d'une procédure identique pour tous les individus. Néanmoins, l'application du protocole a nécessité des choix qui présentent des limites. Ainsi, la matrice proposée aux sujets était toujours identique, avec les attributs en colonne, les offres complètes en ligne, et, contrairement aux recommandations de Dubois (1984), l'ordre à l'intérieur des lignes et colonnes n'a pas subi de rotation systématique. Si ces éléments induisent une bonne validité interne, on peut se demander si une présentation différente, avec les offres en colonne ou les 
marques dans un autre ordre, aurait donné les mêmes résultats. On peut supposer un biais lié à l'habitude de lecture. Autre limite : pour des raisons de durée de l'expérience, il n'a pas été possible de faire un essai à blanc de la méthode ni de débriefing post-expérimental même si les pré-tests en laboratoire ont montré la rapide adaptation des individus à la matrice. La validité externe est également questionnable. Dans un souci de réalisme, la recherche d'information proposée comportait un nombre important d'attributs et de marques. L'expérimentation testée propose alors un choix complexe, où il n'est pas possible de mémoriser toutes les informations disponibles. On peut dès lors s'interroger sur le risque de surinformation (information overlead) et ses conséquences. Notamment, la relation curviligne observée entre l'intensité de la pression temporelle et la vitesse de traitement des attributs est peut-être en lien avec les capacités cognitives limitées identifiées dans le paradigme de surcharge d'information. Cette limite mériterait de plus amples recherches avec un autre produit testé reposant sur un nombre restreint d'attributs. En outre, on peut regretter de ne pas avoir pris en compte des variables explicatives potentielles, notamment le risque perçu et l'implication. Le manque de temps pèse sur la perception du risque, qu'il soit sur les attributs ou exprimé sur le choix final (Volle, 1995). Dès lors, des investigations complémentaires seraient nécessaires pour mieux appréhender les influences conjuguées de la pression temporelle, de l'implication et du risque perçu dans la sélection de certains attributs (hypothèse 3 ).

\section{CONCLUSION}

Cette recherche contribue à une meilleure compréhension des effets de la pression temporelle sur le traitement des informations. D'un côté, un mode de traitement rationnel et logique, caractérisé par un processus de sélection rigoureux, est observé. De l'autre, des effets de seuils entre l'intensité de la pression temporelle et la vitesse de traitement des informations soulignent l'importance de la prise en compte de cette intensité. Le choix a été fait d'examiner ici l'influence de l'intensité de la pression temporelle en comparant des conditions expérimentales sur des individus différents. Toujours dans le domaine des conséquences de la pression temporelle, d'autres voies de recherche pourraient être empruntées. Il serait intéressant, par exemple, de connaître les effets des variations intraconditions. Comment s'organise individuellement l'accélération au fil du temps qui s'écoule ? Ou encore comment la sélection est-elle influencée ? Est-ce par la récence de la dernière information ou davantage par l'effet de primauté ?

Plus généralement, l'impact d'Internet et de tous ses développements suggère de revisiter le thème de la pression temporelle. De nombreux sociologues s'accordent pour reconnaître que l'expérience majeure de notre société contemporaine est l'accélération, fruit d'une accélération technique permise par les nouvelles technologies, d'une accélération sociale et de l'accélération des rythmes de vie (Rosa, 2010). C'est dans ce contexte où « tout devient plus rapide » qu'il convient d'examiner les conséquences de la pression temporelle sur la prise de décision. Le Web, sa puissance, sa vitesse et son immédiateté, a des conséquences considérables sur les processus de décisions impliqués dans tous les domaines, qu'ils soient marchands ou non. On peut alors citer tous les achats de biens et de services en ligne, mais aussi les services culturels comme la musique et les films, directement disponibles et téléchargeables sur Internet.

Enfin, cette recherche plaide pour un renouveau méthodologique. Le thème de l'influence de la pression temporelle sur le traitement des informations a nécessité d'utiliser une méthode appropriée, objective et affranchie des nombreux biais classiques identifiés dans les méthodes classiques. Avec les tables d'information, il n'y a ni biais de mesure verbale, peu de risques de biais de rationalisation ou de conformisme social et pas de biais de verbalisation. Pour certains, la recherche en comportement du consommateur devrait évoluer vers une neuroscience du consommateur (Roullet et Droulers, 2008). Sans aller jusqu'à l'utilisation des méthodes complexes et très spécifiques utilisées en neuro-imagerie et permettant une mesure exacte de l'activité cérébrale, la méthode des tables d'information nous propose une première solution technique, simple et peu coûteuse, éclairant de l'intérieur les séquences du parcours de décision des consommateurs. 


\section{RÉFÉRENCES BIBLIOGRAPHIQUES}

Abendroth L.J. et Diehl K. (2006), Now or never: effects of limited purchase opportunities on patterns of regret over time, Journal of Consumer Research, 33, 3, 342351.

Ackerman D.S. et Gross B.L. (2003), So many choices, so little time: measuring the effects of free choice and enjoyment on perception of free time, time pressure and time deprivation, Advances in Consumer Research, 30, 1, 290-294.

Aggarwal P. et Vaidyanathan R. (2003), Use it or lose it: purchase acceleration affects in time-limited promotions, Journal of Consumer Behavior, 2, 4, 393-403.

Aho K.A. (2007), Acceleration and time pathologies: the critique of psychology in Heidegger's beiträge, Time \& Society, 16, 25-42.

Ariely D. et Wertenbroch K. (2002), Procrastination, deadlines and performance: self-control by precommitment, Psychological Science, 13, 3, 219-224.

Arts N. (2000), Report d'achat et biais cognitifs. Étude de l'effet de cadrage intertemporel, Thèse de doctorat en sciences de gestion, Université des Sciences et Technologies de Lille.

Aubert N. (2003), Le culte de l'urgence : la société malade du temps, Paris, Flammarion.

Beach L.R. (1993), Image theory: an alternative to normative decision theory, Advances in Consumer Research, 20, 1, 235-238.

Benson L. et Beach L.R. (1996), The effects of time constraints on the prechoice screening of decision options, Organizational Behavior \& Human Decision Processes, 67, 2, 222-228.

Ben-Zur H. et Breznitz S.J. (1981), The effect of time pressure on risky choice behavior, Acta Psychologica, 47, $2,89-104$

Bergadaà M. (1988), Le temps et le comportement de l'individu, $1^{\text {re }}$ partie, Recherche et Applications en Marketing, 3, 4, 57-72.

Bergadaà M. (1989), Le temps et le comportement de l'individu, $2^{\mathrm{e}}$ partie, Recherche et Applications en Marketing, 4, 1, 37-55.

Bergadaà M. (2005), Le temps-cadre de l'action du shopper : la coexistence de quatre modèles actuels et leur impact sur le positionnement des enseignes, Actes de la conférence Temps et comportement du consommateur, IUT de Lille.

Bergadaà M. (2007), Temporal frameworks and individual cultural activities: four typical profiles, Time \& Society, 16, 387-407.

Bergadaà M. et Coraux G. (2004), L'attitude à l'égard d'Internet, du shopping, et le comportement des Internautes, cahiers de recherche, École HEC, Genève.

Bergson H. (1922), Durée et simultanéité. À propos de la théorie d'Einstein, Paris, PUF.
Berry L.L. (1979), The time-buying consumer, Journal of Retailing, 55, 4, 58-69.

Bettman J.R., Luce M.F. et Payne J.W. (1998), Constructive consumer choice processes, Journal of Consumer Research, 25, 3, 187-217.

Bouder-Pailler D. (2003), La conception du temps du consommateur influence-t-elle les comportements d'achat sur Internet ? Propositions de mesures et modèle, Revue Française du Marketing, 191, 81-99.

Brannon L.A. et Brock T.C. (2001), Limiting time for responding enhances behavior corresponding to the merits of compliance appeals: refutations of heuristiccue theory in service and consumer settings, Journal of Consumer Psychology, 10, 3, 135-146.

Chien-Huang L. et Pei-Hsun W. (2005), How to deal with conflicts? The effect of consumers' subjective time pressure on product attitude judgment and choice, The Journal of American Academy of Business, 6, 1, 219224.

Chu P.C. et Spires E.E. (2001), Does time constraint on users negate the efficacy of decision support systems?, Organizational Behavior \& Human Decision Processes, 85, 2, 226-249.

Comm C. et Palachek A.D. (1984), The effect of various waiting line times and regret levels on individual consumption time, Advances in Consumer Research, $11,41-45$.

Cotte J. et Ligas M. (2003), Timestyle and shopping style, European Advances in Consumer Research, 6, 89-95.

Cotte J. et Ratneshwar S. (1998), Consumer decisions on discretionary time: a sociocognitive perspective, Advances in Consumer Research, 25, 268-275.

Darpy D. (1999), La procrastination du consommateur : une contribution à l'explication du report d'achat, Thèse de doctorat en sciences de gestion, Université de Paris IX - Dauphine

Des Garets V. (1999), Études et recherches commerciales, Paris, Economica.

Dhar R. (1997), Consumer preference for a no-choice option, Journal of Consumer Research, 24, 2, 215-231.

Dhar R. et Nowlis S.M. (1999), The effect of time pressure on consumer choice deferral, Journal of Consumer Research, 25, 4, 369-384.

Dhar R., Nowlis S.M. et Sherman S.J. (2000), Trying hard or hardly trying: an analysis of context effects in choice, Journal of Consumer Psychology, 9, 4, 189-200.

Dubois B. (1984), Les tables d'information : potentiel et limites, Revue Française du Marketing, 97, 3-16.

Durrande-Moreau A. (1994), Qualité de service et perception du temps, Thèse de doctorat en sciences de gestion, Université Pierre-Mendès-France, Grenoble.

Edland A. (1993), The effects of time pressure on choices and judgments of candidates to an university program, in A. Edland et O. Svenson (coord.), Time pressure and stress in human judgment and decision making, New York, Plenum, 145-156.

Edland A. et Svenson O. (coord.) (1993), Judgment and decision making under time pressure: studies and findings, Time pressure and stress in human judgment and decision making, New York, Plenum, 27-40. 
Evrard Y., Pras B. et Roux E. (1993), Market : Études et recherches en marketing, Paris, Nathan.

Feldman L.P. et Hornik J. (1981), The use of time: an integrated conceptual model, Journal of Consumer Research, 7, 4, 407-419.

Greenleaf E.A. et Lehmann D.R. (1995), Reasons for substantial delay in consumer decision making, Journal of Consumer Research, 22, 2, 186-199.

Gross B.L. (1994), Consumer responses to time pressure: a qualitative study with homeowners in foreclosure, Advances in Consumer Research, 21, 120-125.

Hall E.T. (1959), The silent language, Garden City, New York, Double day \& Company.

Hauser J.R., Urban G.L. et Weinberg B.D. (1993), How consumers allocate their time when searching for information, Journal of Marketing Research, 30, 4, 452466.

Hawes D. (1980), The time variable in models of consumer behavior, Advances in Consumer Research, 7, 1, 442447.

Hendrix P.E. (1984), Antecedents and consequences of time use: proposed measures and preliminary evidence, Advances in Consumer Research, 11, 35-40.

Hendrix P.E. et Martin C.R. (1981), Temporal incongruency in consumer behavior, Advances in Consumer Research, 8, 182-186.

Herrington J.D. et Capella L.M. (1995), Shopper reactions to perceived time pressure, International Journal of Retail \& Distribution Management, 23, 12, 13-21.

Hornik J. (1984), Subjective vs. objective time measures: a note on the perception of time in consumer behavior, Journal of Consumer Research, 11, 1, 615-618.

Hornik J. (1993), The role of affect in consumers' temporal judgements, Psychology \& Marketing, 10, 3, 239-255.

Hui M.K., Tse A.C. et Zhou L. (2006), Interaction between two types of information on reactions to delay, Marketing Letters, 17, 151-162.

Jacoby J., Chestnut R.W. et Fisher W.A. (1978), A behavioral process approach to information acquisition in nondurable purchasing, Journal of Marketing Research, 15, 4, 532-544.

Jacoby J., Jaccard J., Currim I., Kuss A., Ansari A. et Troutman T. (1994), Tracing the impact of item-byitem information accessing on uncertainty reduction, Journal of Consumer Research, 21, 2, 291-303.

Jewel R.D. (2003), The effects of deadline pressure on attitudinal ambivalence, Marketing Letters, 14, 2, 83-95.

Kang Y.S., Herr P.M et Page C.M. (2003), Time and distance: asymmetries in consumer trip knowledge and judgments, Journal of Consumer Research, 30, 3, 420429.

Kaufman C. et Lane P.M. (1990), The intentions and extensions of the time concept: contributions from a sociological perspective, Advances in Consumer Research, 17, 895-901.

Kellaris J.J. et Mantel S.P. (1994), The influence of mood and gender on consumers' time perception, Advances in Consumer Research, 21, 514-518.
Kerstholt J.H. (1994), The effect of time pressure on decision-making behaviour in a dynamic task environment, Acta Psychologica, 86, 1, 89-104.

Kim H.Y. et Kim Y.K. (2008), Shopping enjoyment and store shopping modes: the moderating influence of chronic time pressure, Journal of Retailing and Consumer Services, 15, 410-419.

Koiso-Kanttila N. (2005), Time, attention, authenticity and consumer benefits of the Web, Business Horizon, 48, 63-70.

Kolodinsky J. (1990), Time as a direct source of utility: the case of price information search for groceries, Journal of Consumer Affairs, 24, 1, 89-109.

Leclerc F., Schmitt B.H. et Dubé L. (1995), Waiting time and decision making: is time like money, Journal of Consumer Research, 22, 1, 110-119.

Linder S. (1970), The harried leisure class, New York, Columbia University Press.

Mantel S.P. et Kellaris J.J. (2003), Cognitive determinants of consumers' time perceptions: the impact of resources required and available, Journal of Consumer Research, $29,4,531-538$.

Marmorstein H. (1992), The value of time in price-comparison shopping: survey and experimental evidence, Journal of Consumer Research, 19, 1, 52-61.

Maule A.J., Hockey G.R. et Bdzola L. (2000), Effects of time-pressure on decision-making under uncertainty: changes in affective state and information processing strategy, Acta Psychologica, 1, 104, 283-301.

Miller J.G. (1960), Information input and psychopathology, American Journal of Psychiatry, 116, 695-704.

Miyazaki A.D. (1993), How many shopping days until christmas? A preliminary investigation of time pressures, deadlines and planning levels on holiday gift purchases, Advances in Consumer Research, 20, 331335.

Nelmapius A.H., Boschoff C., Calitz A.P. et Klemz B.R. (2005), The impact of the information search variables, time pressure and involvement, on buying behaviour in a three-dimensional hypermedia computer-mediated environment, South African Journal of Business Management, 36, 3, 1-13.

Nickols S.Y. et Fox K.D. (1983), Buying time and saving time: strategies for managing household production, Journal of Consumer Research, 10, 2, 197-208.

Okada E.M. et Hoch S.J. (2004), Spending time versus spending money, Journal of Consumer Research, 31, 2, 313-323.

Park C.W., Iyer E.S. et Smith D.C. (1989), The effects of situational factors on in-store grocery shopping behavior: the role of store environment and time available for shopping, Journal of Consumer Research, 15, 4, 422-433.

Payne J.W., Bettman J.R. et Johnson E.J. (1993), The adaptive decision maker, Cambridge, University Press.

Payne J.W. (1976), Task complexity and contingent processing in decision making: an information search and protocol analysis, Organizational Behavior and Human Performance, 16, 366-387. 
Payne J.W., Bettman J.R. et Johnson E.J. (1988), Adaptive selection in decision making, Journal of Experimental Psychology: Learning, Memory and Cognition, 14, 3, 534-552.

Payne J.W., Bettman J.R. et Luce M.F. (1996), When time is money: decision behavior under opportunity-cost time pressure, Organizational Behavior and Human Decision Processes, 66, 2, 131-152.

Petr C. et Hess-Miglioretti A. (2010), La méthode des tables d'information : un renouvellement grâce à Internet ?, Décisions Marketing, 57, 19-30.

Pieters R., Warlop L. et Hartog M. (1997), The effect of time pressure and task motivation on visual attention to brands, Advances in Consumer Research, 24, 281-287.

Reeves J.B. et Szafran R.F. (1996), For what and for whom do you need more time, Time \& Society, 5, 237-251.

Riveline C. (1991), De l'urgence en gestion, Paris, Annales des Mines.

Rizkalla A.N. (1989), Sense of time urgency and consumer well-being: testing alternative causal models, Advances in Consumer Research, 16, 1, 180-188.

Robinson J.P. et Nicosia F.M (1991), Of time, activity, and consumer behavior: an essay on findings, interpretations, and needed research, Journal of Business Research, 22, 2, 171-187.

Rosa H. (2010), Accélération, une critique sociale du temps, Paris, La Découverte.

Roullet B. et Droulers O. (2008), Neuroscience du consommateur : rupture paradigmatique ?, Actes $d u 18^{e}$ Congrès AFM, Vincennes.

Schellinck D.A. (1983), Cue choice as a function of time pressure and perceived risk, Advances in Consumer Research, 10, 470-475.

Schurr P.H. et Brucks M. (1991), Deal search: an approach for computer-controlled information processing experiments involving bargainable attributes, Advances in Consumer Research, 18, 1, 591-596.

Sullivan O. (2008), Busyness, status distinction and consumption strategies of the income rich, time poor, Time \& Society, 2008, 17, 5-26.

Suri R. et Monroe K.B. (2003), The effects of time constraints on consumers' judgments of price and products, Journal of Consumer Research, 30, 1, 92-104.

Svenson O. et Benson A.J. (1993), Framing and time pressure in decision making, in A. Edland et O. Svenson (coord.), Time pressure and stress in human judgment and decision-making, New York, Plenum, 133-144.

Svenson O., Edland A. et Slovic P. (1990), Choices and judgments of incompletely described decision alternatives under time pressure, Acta Psychologica, 75, 2, 153-169.
Szollos A. (2009), Toward a psychology of chronic time pressure: conceptual and methodological review, Time \& Society, 18, 332-350.

Topi H., Valacich J. et Hoffer J.A. (2005), The effects of task complexity and time in availability limitations on human performance in database query tasks, International Journal of Human-Computer Studies, 62, 3, 349-379.

Urien B. (1994), Tentative d'interprétation temporelle du comportement exploratoire du consommateur, Thèse de doctorat en sciences de gestion, Université de Rennes 1

Urien B. (2007), Selfless time: from life to death anticipation, Time \& Society, 16, 367-386.

Usunier J.-C. (1995), Une critique de la fonctionnalité de l'urgence, Temporalistes, 29, 5-10.

Usunier J.-C. et Valette Florence P. (2007), The time styles scale: a review of developments and replications over 15 years, Time \& Society, 16, 333-366.

Valette-Florence P., Usunier J.-C., Ferrandi M. et Roehrich G. (2001), An exploratory study of the links between personal values and temporal orientations, Asia Pacific, Advances in Consumer Research, 4, 37-45.

Verplanken B. (1993), Need for cognition and external information search: responses to time pressure during decision making. Journal of Research in Personality, 27, 238-252.

Volle P. (1995), Le concept de risque perçu en psychologie du consommateur : antécédents et statut théorique, Recherche et Applications en Marketing, 10, 1, 39-56.

Voss J. et Blackwell R.D. (1975), Market for leisure time, Advances in Consumer Research, 2, 837-846.

Weenig M.W.H. et Maarleveld M. (2002), The impact of time constraint on information search strategies in complex choice tasks, Journal of Economic Psychology, 23, 689-702.

Wright P. (1974), The harassed decision-maker-time pressures, distractions, and the use of evidence, Journal of Applied Psychology, 59, 5, 555-561.

Wright P. et Weitz B. (1977), Time horizon effects on product evaluation strategies, Journal of Marketing Research, 14, 4, 429-443.

Zakay D. (2005), Attention et jugement temporel, Psychologie Française, 50, 65-70.

Zakay D. et Wooler S. (1984), Time pressure, training and decision effectiveness, Ergonomics, 27, 3, 273-284. 